\title{
Indirect constraints on the scalar di-photon resonance at the LHC
}

\author{
Florian Goertz, ${ }^{a}$ Jernej F. Kamenik, ${ }^{a, b, c}$ Andrey Katz ${ }^{a, d}$ and Marco Nardecchia ${ }^{e}$ \\ a Theory Division, CERN, 1211 Geneva 23, Switzerland \\ ${ }^{b}$ Jožef Stefan Institute, Jamova 39, 1000 Ljubljana, Slovenia \\ ${ }^{c}$ Faculty of Mathematics and Physics, University of Ljubljana, \\ Jadranska 19, 1000 Ljubljana, Slovenia \\ ${ }^{d}$ Department of Theoretical Physics and Center for Astroparticle Physics, Université de Genève, \\ 24 quai E. Ansermet, CH-1211, Geneva 4, Switzerland \\ ${ }^{e}$ DAMPT, University of Cambridge, \\ Wilberforce Road, Cambridge CB3 0WA, United Kingdom \\ E-mail: florian.goertz@cern.ch, jernej.kamenik@cern.ch, \\ andrey.katz@cern.ch, m.nardecchia@damtp.cam.ac.uk
}

AbSTRACT: Motivated by the tantalizing excesses recently reported in the di-photon invariant mass spectrum at the LHC, we scrutinize some implications of scalar di-photon resonances in high energy proton-proton collisions. In particular, indications of a large width impose several challenges for model building. We show how calculability and unitarity considerations severely limit possible perturbative realizations of such a signal and propose a simple criterion that can be adapted to any renormalizable model. Furthermore, we discuss correlations between a di-photon excess and precision observables, including the anomalous magnetic and electric dipole moments of quarks and leptons, neutral meson oscillations and radiative flavor changing neutral current mediated decays of heavy leptons and hadrons. We find that existing searches and measurements significantly constrain the possibilities for a scalar resonance decaying into final states involving Standard Model fermions. We propose future search strategies which could elucidate some remaining currently unconstrained decay channels and discuss possible correlations between the diphoton excess and several recently reported flavor anomalies, showing that the latter can be addressed in a new incarnation of a gauged $\mathrm{U}(1)^{\prime}$ model, with the di-photon resonance being the physical remnant of the $\mathrm{U}(1)^{\prime}$-breaking field.

Keywords: Beyond Standard Model, Effective field theories, Renormalization Group

ARXIV EPRINT: 1512.08500 


\section{Contents}

1 Introduction 1

$\begin{array}{llll}2 & \text { Setup } & 4\end{array}$

3 Calculability in models of weakly coupled new fermions 5

4 Constraints on couplings to SM fermions $\quad 12$

$\begin{array}{lll}4.1 & \text { Dipole moments } & 12\end{array}$

$\begin{array}{lll}4.2 & \text { Flavor observables } & 18\end{array}$

4.3 Implications of $S \rightarrow \gamma \gamma$ for $R_{K}$ puzzle $\quad 21$

5 Conclusions $\quad 24$

\section{Introduction}

Very recently, ATLAS reported an excess in the search for resonances decaying into photon pairs around a mass of $M_{\gamma \gamma} \sim 750 \mathrm{GeV}$, using the first $3.2 \mathrm{fb}^{-1}$ of data at $\sqrt{s}=13 \mathrm{TeV}$ collected in run II at the LHC. The local (global) significance of the excess is $3.9 \sigma(2.3 \sigma)$, taking the best-fit value for the width of the resonance of $\Gamma \sim 45 \mathrm{GeV}$ [1]. Interestingly, the signal is in tentative agreement with results from the similar CMS search, which sees a local excess of $2 \sigma$ for the same width, employing $2.6 \mathrm{fb}^{-1}$ of data [2]. ${ }^{1}$ There were also less significant upward fluctuations in this mass region in the run I data collected at $8 \mathrm{TeV}[3,4]$. The surplus might be just a statistical fluctuation, however it could also be the first hint and guidance to physics beyond the Standard Model (SM). While there have been in fact immediately numerous attempts to explain this anomaly in terms of a new particle in different concrete scenarios beyond the SM [5-76]. Here we want to take a rather general perspective and relate the excess to other observables and considerations, which can provide consistency and phenomenological constraints on its explanations.

Since the tentative new state (which we henceforth denote by $S$ ) couples to two photons it can either have spin zero or spin two, and in the present work we will focus on the former case. Moreover, the new state can be produced singly or associated with other final states. Currently there are no experimental indications of significant additional electromagnetic or hadronic activity in signal events, and no significant missing transverse energy has been reported. All together this prefers prompt single production as the simpler explanation, which we will also concentrate on here. Theoretically, the resonance could be a singlet under the SM gauge symmetry or part of a larger electroweak multiplet. While we will mostly focus on the former case, we will comment on generalizing our results to the possibility

\footnotetext{
${ }^{1}$ For a narrow width, which seems to be preferred in CMS, the significance increases to $2.6 \sigma$.
} 
that the state carries non-zero weak isospin. Finally, consistency of the run II excesses with run I data requires the production cross section to increase between $8 \mathrm{TeV}$ and $13 \mathrm{TeV}$ proton-proton collisions by at least a factor five, indicating production from gluon fusion or non-valence $(b, c, s)$ quark annihilation [77]. In the present work we will focus on the first possibility, however many of our results can be easily generalized to the other cases.

As we will show in section 2 (and has been stressed in some of the existing literature, cf. [77]) the observed signal strengths imply uncomfortably large couplings of $S$ to photons (and gluons). While numerous perturbative model realizations have already been proposed, they generically require the existence of large multiplicities or (color) charges of additional new massive particles (mediators), preferably close to the TeV scale. From a model building point of view, strongly coupled extensions of the SM thus seem to be preferred. Here we want to support and reinforce this argument showing that various supposedly renormalizable weakly coupled extensions are de facto not calculable at all. While we cannot exclude that perturbation theory in any "weakly coupled" model breaks down at the mass-scale of the mediators, we show with a concrete toy model capturing the essential ingredients of most existing proposals, that the problem of calculability is indeed very severe.

Various authors have suggested models with couplings of order unity. Naively one could think that this is enough to guarantee a perturbative expansion of the theory, however, in order to fit the phenomenological data, a plethora of new states that carry electric charge (to generate the decay into photons) and color charge (in order for example to have a sizable production cross section due to the interactions with the gluons in the proton) have to be added to the theory. However, the large amount of new states affects the predictivity of the theory in two ways:

- The new states modify the running of the couplings inducing the possibility of the appearance of Landau poles in the ultraviolet (UV). If we do not insist on having a calculable model that can be extrapolated to very high energies this is not a serious issue: perturbative expansion is valid, albeit only in a limited range of energy scales;

- The new dynamics could be such that perturbation theory breaks down already at the matching scales of the mediators, i.e. because the relevant expansion parameter the 't Hooft coupling - grows non-perturbative. If this is the case any computation is not reliable.

It is therefore important to find an easy adaptable criterion to understand if a given model is calculable or not. In section 3 we propose exactly one such criterion and examine it on an explicit concrete example involving new color and/or electromagnetically (EM) charged vector-like fermions with Yukawa-like couplings to $S$.

Once/if the $S$ signal is established, it will also be of utmost importance to study its properties and interactions with SM degrees of freedom. Especially because the large decay width, favored by the ATLAS measurements, could indicate $S$ decay channels beyond di-photons. Fortunately, precision low energy measurements already provide abundant information in the form of constraints on the potential couplings of $S$. For example, if $S$ couples to SM quark anti-quark pairs of different generations, it will induce contributions 
to local four-Fermi interactions at low energies which can be probed with neutral meson oscillation measurements. In addition, its sizable coupling to photons (and gluons) generically predicts the appearance of (chromo) magnetic and electric dipole operators, which are tightly constrained by existing low energy measurements and searches. In particular, if the products of couplings of $S$ to pairs of photons (gluons) and (colored) SM fermions $f$ violate $\mathrm{CP}$, they will induce a (chromo) electric dipole moment $((\mathrm{C}) \mathrm{EDM})$ of $f$ at the one-loop order. The CP conserving combination on the other hand generates the corresponding anomalous (chromo) magnetic dipole moment ((C)MDM). Finally, if $S$ couples to SM fermions of different generations, it will induce radiative flavor changing neutral current (FCNC) or lepton flavor violating (LFV) transitions, both of which are already tightly constrained by experiments. We explore the implications of these low energy constraints on the collider phenomenology of $S$ in section 4 .

In few cases we also have weak hints for possible BSM physics which can also potentially be connected to the di-photon excess. Therefore among the various observables we devote special attention to two where experimental measurements have been exhibiting persistent tensions with SM estimates and which can be naturally linked to the existence of a heavy scalar resonance decaying to photon pairs:

1. The anomalous magnetic moment of the muon $\left(a_{\mu}\right)$

2. The measurements of rare semileptonic $B$ meson decays $B \rightarrow K^{(*)} \ell^{+} \ell^{-}$.

For the former we will show that models addressing the anomaly via loop diagrams involving $S$ are in general tightly constrained from limits on $S \rightarrow \mu^{+} \mu^{-}$decays, while for the latter we will present and constrain a simple solution with a gauged $\mathrm{U}(1)^{\prime}$ symmetry, where the di-photon resonance is the physical remnant of the $\mathrm{U}(1)^{\prime}$-breaking field.

At this point we note that several previous works have considered flavor constraints on the $750 \mathrm{GeV}$ diphoton resonance. In particular, refs. [15, 30] have studied possible implications of the $750 \mathrm{GeV}$ resonances on the $(g-2)_{\mu}$ within particular models that they have analyzed. Similarly, refs. $[17,22]$ made an attempt to explain the data in conjunction with the $R_{K}$ and $R_{D^{*}}$ measurements, while [19] tried to connect it to the $t \bar{t}$ forward-backward asymmetry. However, we emphasize that this work is the first attempt to consider the implications of the di-photon resonance on flavor physics in relatively modelindependent way, with generic assumptions that we outline in section 2. Compared the previous works we also consider a much broader spectrum of flavor observables to constrain the couplings of the new resonance to SM fermions.

To summarize the structure of the remainder of the paper: the basic experimental facts on $S$ production and decays at the LHC are reported in section 2, where we also define the theoretical framework within which our conclusions are derived. This is followed by the discussion on the calculability of weakly coupled UV realizations of the effective low energy interactions of $S$ in section 3. The constraints and implications of $S$ couplings to SM fermions are examined in section 4 . We conclude with a summary of our results and discussion of possible future extensions, presented in section 5 . 


\section{Setup}

We follow ref. [77] in parametrizing the signal strength of $S$ production through gluon fusion and its decays to pairs of photons in terms of the corresponding $S$ decay widths $\Gamma_{\gamma \gamma} \equiv \Gamma(S \rightarrow \gamma \gamma)$ and $\Gamma_{g g} \equiv \Gamma(S \rightarrow g g)$ computed at leading order in QCD. Assuming $\sigma(p p \rightarrow S) \times \mathcal{B}(S \rightarrow \gamma \gamma) \approx(6.0 \pm 1.5) \mathrm{fb}$ at $\sqrt{s}=13 \mathrm{TeV}$ LHC, the current data are reproduced for

$$
\frac{\Gamma_{\gamma \gamma} \Gamma_{g g}}{M \Gamma} \approx 8 \times 10^{-7} .
$$

where $\Gamma \equiv \sum_{f} \Gamma(S \rightarrow f)$ is the total $S$ width and $M \simeq 750 \mathrm{GeV}$ its mass. In addition, the ATLAS data prefer

$$
\frac{\Gamma}{M} \approx 0.06
$$

At any scale $\mu_{0}$ above $M$ one can parametrize the relevant $S$ interactions to photons and gluons in terms of local effective operators ${ }^{2}$

$$
\mathcal{L}_{F}=-\frac{\epsilon_{\gamma} e^{2} S F^{2}}{2 \Lambda_{\gamma}}-\frac{\epsilon_{g} g_{s}^{2} S G^{2}}{2 \Lambda_{g}}-\frac{\tilde{\epsilon}_{\gamma} e^{2} S F \tilde{F}}{2 \tilde{\Lambda}_{\gamma}}-\frac{\tilde{\epsilon}_{g} g_{s}^{2} S G \tilde{G}}{2 \tilde{\Lambda}_{g}}
$$

which encode the contributions from all degrees of freedom with masses above $\mu_{0}$. We will also assume that the $S$ field does not condense which is equivalent to requiring that the particle $S$ is an excitation around a minimum of the scalar potential. We can then identify $\Lambda_{i}$ with the mass of the lowest lying state generating the terms in $\mathcal{L}_{F}$ and absorb additional dependence on the couplings, charges and multiplicities of such states into $\epsilon_{i}$. In absence of additional contributions to $\Gamma_{\gamma \gamma}$ and $\Gamma_{g g}$ and focusing for the moment on CP even interactions, the product $\left(\epsilon_{\gamma} / \Lambda_{\gamma}\right)\left(\epsilon_{g} / \Lambda_{g}\right)$ is bounded from below from (2.1), leading to ${ }^{3}$

$$
\epsilon_{\gamma} \epsilon_{g} \gtrsim 0.06 \times \frac{\Lambda_{\gamma} \Lambda_{g}}{(1 \mathrm{TeV})^{2}} \sqrt{\frac{\Gamma / M}{0.06}}
$$

where the width is in general bounded from below by

$$
\frac{\Gamma}{M} \geq \pi\left(\alpha^{2} \epsilon_{\gamma}^{2} \frac{M^{2}}{\Lambda_{\gamma}^{2}}+8 \alpha_{s}^{2} \epsilon_{g}^{2} \frac{M^{2}}{\Lambda_{g}^{2}}\right)
$$

If however $S$ couples to (in particular charged and/or colored) particles $\mathcal{Q}$ with masses below $\mu_{0}$, these have to be included in the theory. Considering for concreteness contributions due to couplings to fermions (generalization to the case of scalars or even vectors is straightforward), the relevant interactions are of the form

$$
\mathcal{L}_{Y}=-\sum_{\mathcal{Q}} Y_{\mathcal{Q}} S \overline{\mathcal{Q}} P_{L} \mathcal{Q}+\text { h.c. }+\ldots
$$

\footnotetext{
${ }^{2}$ Far above the electroweak symmetry breaking scale, one should replace the first term with its $\mathrm{SU}(2)_{L} \times$ $\mathrm{U}(1)_{Y}$ invariant versions. However, this distinction has no barring on our subsequent discussion and results.

${ }^{3}$ Completely analogous expressions can be derived for CP odd terms proportional to $\tilde{\epsilon}_{\gamma}$ and $\tilde{\epsilon}_{g}$ as well as possible mixed terms .
} 
where $P_{L} \equiv\left(1-\gamma_{5}\right) / 2$. The dots denote additional possible terms coupling different $\mathcal{Q}$ to $S$, which however do not contribute to $\Gamma_{g g}$ and $\Gamma_{\gamma \gamma}$ at one loop order, as well as terms suppressed by the cut-off scale of the theory. Written in the mass basis, the sum over $\mathcal{Q}$ might include SM fermions. Then $\Gamma_{\gamma \gamma}$ and $\Gamma_{g g}$ can be written as

$$
\begin{aligned}
& \frac{\Gamma_{\gamma \gamma}}{M}=\pi \alpha^{2}\left(\epsilon_{\gamma}^{2} \frac{M^{2}}{\Lambda_{\gamma}^{2}}+\tilde{\epsilon}_{\gamma}^{2} \frac{M^{2}}{\tilde{\Lambda}_{\gamma}^{2}}+\left|\frac{1}{4 \pi^{2}} \sum_{\mathcal{Q}} d_{\mathcal{Q}} Q_{\mathcal{Q}}^{2} \operatorname{Re}\left(Y_{\mathcal{Q}}\right) \mathcal{F}_{\mathcal{Q}}^{\operatorname{Re}}\left(2 M_{\mathcal{Q}} / M\right)\right|^{2}+(\operatorname{Re} \rightarrow \operatorname{Im})\right) \\
& \frac{\Gamma_{g g}}{M}=8 \pi \alpha_{s}^{2}\left(\epsilon_{g}^{2} \frac{M^{2}}{\Lambda_{g}^{2}}+\tilde{\epsilon}_{g}^{2} \frac{M^{2}}{\tilde{\Lambda}_{g}^{2}}+\left|\frac{1}{4 \pi^{2}} \sum_{\mathcal{Q}} C_{\mathcal{Q}} \operatorname{Re}\left(Y_{\mathcal{Q}}\right) \mathcal{F}_{\mathcal{Q}}^{\operatorname{Re}}\left(2 M_{\mathcal{Q}} / M\right)\right|^{2}+(\operatorname{Re} \rightarrow \operatorname{Im})\right)
\end{aligned}
$$

where $d_{\mathcal{Q}}$ is the dimension of the QCD representation of $\mathcal{Q}$ and $C_{\mathcal{Q}}$ its index while $Q_{\mathcal{Q}}$ is the EM charge of $\mathcal{Q}$. The functions $\mathcal{F}_{\mathcal{Q}}^{\mathrm{Re}, \operatorname{Im}}(x)$ depend only on the spin of $\mathcal{Q}$. They are resonantly enhanced at $x \sim 1$ and decouple at large and small $x$ as $\mathcal{F}_{\mathcal{Q}}^{\mathrm{Re}, \operatorname{Im}}(x) \propto 1 / x$ and $\mathcal{F}_{\mathcal{Q}}^{\operatorname{Re}, \operatorname{Im}}(x) \propto x \log (x)$, respectively. Due to this decoupling nature, we can without loss of generality take $\mu_{0} \sim M$ in which case the sums only run over SM fermions and possibly a few additional states with masses below $M$ while the rest is encoded in the coefficients $\epsilon_{i}$. All SM - except possibly the top quark - contributions are also completely negligible. We immediately see why the large required radiative widths are not easily reproduced in perturbative models. In fact, as shown in [77], reproducing the data via loops of massive particles coupling to $S$ requires either large multiplicities or large $Y$, charges and color representations. We study this issue quantitatively in the next section.

\section{Calculability in models of weakly coupled new fermions}

To start our discussion, we will introduce a criterion to check if a given model is calculable. Assuming that a model contains a series of couplings $y_{i}$, then, inspired by the $1 / 16 \pi^{2}$ suppression in the loop expansion, one could suggest that a plausible criterion is the requirement that $\left|y_{i}\right|<4 \pi$ for every coupling in the theory. However, considering the New Physics models that aim at explaining the di-photon resonance, this proposal could be misleading. If there are $N$ copies of mediators propagating in the loop, it is clear that the multiplicity of the states has to be considered in the criterion. For example the requirement $\left|y_{i}\right|<\frac{4 \pi}{\sqrt{N}}$, à la 't Hooft, could be more meaningful than $\left|y_{i}\right|<4 \pi$.

We can try to improve the definition of our criterion in the following way. The value of the coupling $y_{i}$ depends on the energy scale $\mu$ and its rate of change with energy is dictated by the beta function $\beta_{y_{i}}$. If we change the energy scale by an amount $\delta \mu$ we get a variation to the coupling $y_{i}$ that amounts to

$$
\delta y_{i} \approx \frac{\partial y_{i}}{\partial \mu} \delta \mu
$$


The relative variation is given by

$$
\frac{\delta y_{i}}{y_{i}} \approx \frac{\mu}{y_{i}} \frac{\partial y_{i}}{\partial \mu} \frac{\delta \mu}{\mu}=\frac{\beta_{y_{i}}}{y_{i}} \frac{\delta \mu}{\mu}
$$

We conclude that if the the ratio $\left|\beta_{y_{i}} / y_{i}\right|$ is large, the coupling constant varies a lot even with small changes of the renomalization scale and henceforth one runs into troubles with calculability. Based on this, our final proposal for the criterion is

$$
\left|\frac{\beta_{y_{i}}^{(1)}(\{y\})}{y_{i}}\right|<1 \quad \text { for every coupling } y_{i}
$$

where $\beta_{y_{i}}^{(1)}$ is the $\beta$ function of the coupling $y_{i}$ truncated at the one-loop level. The $\beta$ functions are obtained as the sum of various contributions, so avoiding accidental cancellation, we will apply the proposed bound (3.3) to every contribution. This criterion benefits also from the fact that the one-loop beta functions for renormalizable gauge-Yukawa theories are well known and easy to compute (actually complete formulae are available up to two loops [78-81]).

We will now analyze the restriction imposed by (3.3) on a simple toy model. Despite its simplicity, our toy model captures the main features of several proposals which have appeared in the literature. Only in this section, we use a two-component notation for fermions. We introduce $N_{Q}$ copies of neutral vector-like QCD triplets $\left(Q_{A}, Q_{A}^{c}\right)$ as well as $N_{E}$ copies of colorless vector-like fermions $\left(E_{B}, E_{B}^{c}\right)$, singlet under $\mathrm{SU}(2)_{L}$ and with hypercharge $Y$. We assume the theory to be invariant under a $\mathrm{SU}\left(N_{Q}\right) \times \operatorname{SU}\left(N_{E}\right)$ global symmetry. The $750 \mathrm{GeV}$ resonance is represented by a real pseudo-scalar field $S$. We also impose invariance under parity transformation. In two-component notation the transformation rules that we assume are

$$
\begin{aligned}
Q_{A}(\mathbf{x}, t) & \rightarrow\left(Q_{A}^{c}(-\mathbf{x}, t)\right)^{\dagger}, \\
E_{B}(\mathbf{x}, t) & \rightarrow\left(E_{B}^{c}(-\mathbf{x}, t)\right)^{\dagger}, \\
S(\mathbf{x}, t) & \rightarrow-S(-\mathbf{x}, t) .
\end{aligned}
$$

The most general Lagrangian beyond the SM with the above assumed symmetries is given by

$$
\begin{aligned}
\mathcal{L}_{\mathrm{NP}}= & i Q_{A}^{\dagger} \sigma^{\mu} D_{\mu} Q_{A}+i Q_{A}^{c \dagger} \sigma^{\mu} D_{\mu} Q_{A}^{c}+i L_{B}^{\dagger} \sigma^{\mu} D_{\mu} L_{B}+i L_{B}^{c \dagger} \sigma^{\mu} D_{\mu} L_{B}^{c} \\
& -\left(M_{Q} Q_{A}^{c} Q_{A}+M_{E} E_{B}^{c} E_{B}+i y_{q} Q_{A}^{c} Q_{A} S+i y_{e} E_{B}^{c} E_{B} S+\text { h.c. }\right) \\
& -\left(\frac{M^{2}}{2} S^{2}+\frac{\lambda}{4 !} S^{4}\right) .
\end{aligned}
$$

Invariance under parity forces $y_{q}$ and $y_{e}$ to be real and it is responsible also for the absence of linear and cubic terms in $S$ in the potential. We are also omitting for simplicity the scalar quartic coupling $H^{\dagger} H S^{2}$. 
In order to apply our criterion, we need to compute one-loop $\beta$ functions of the couplings $g_{s}, g^{\prime}, y_{q}, y_{e}$ and $\lambda$. Using the analytic expressions from [81], we obtain:

$$
\begin{aligned}
\beta_{g_{s}} & =\frac{g_{s}^{3}}{16 \pi^{2}}\left(7-\frac{2}{3} N_{Q}\right), \\
\beta_{g^{\prime}} & =\frac{g^{\prime 3}}{16 \pi^{2}}\left(\frac{41}{6}+\frac{4}{3} N_{E} Y^{2}\right), \\
\beta_{y_{q}} & =\frac{y_{q}}{16 \pi^{2}}\left(3 y_{q}^{2}+2\left(3 N_{Q} y_{q}^{2}+N_{E} y_{e}^{2}\right)-8 g_{s}^{2}\right), \\
\beta_{y_{e}} & =\frac{y_{e}}{16 \pi^{2}}\left(3 y_{e}^{2}+2\left(3 N_{Q} y_{q}^{2}+N_{E} y_{e}^{2}\right)-\frac{18}{5} g^{\prime 2} Y^{2}\right), \\
\beta_{\lambda} & =\frac{1}{16 \pi^{2}}\left[3 \lambda^{2}+8\left(3 N_{Q} y_{q}^{2}+N_{E} y_{e}^{2}\right) \lambda-48\left(3 N_{Q} y_{q}^{4}+N_{E} y_{e}^{4}\right)\right] .
\end{aligned}
$$

From the $\beta$ functions of the gauge couplings we get:

$$
\begin{aligned}
& \left|\frac{\beta_{g_{s}}}{g_{s}}\right|<1 \Longrightarrow N_{Q}<\frac{3}{2}\left(\frac{4 \pi}{\alpha_{s}}+7\right) \approx 200, \\
& \left|\frac{\beta_{g^{\prime}}}{g^{\prime}}\right|<1 \Longrightarrow N_{E} Y^{2}<\frac{3}{4}\left(\frac{4 \pi}{\alpha^{\prime}}-\frac{41}{6}\right) \approx 1580 .
\end{aligned}
$$

The numerical approximations are obtained assuming $\alpha_{s}=0.1$ and $\alpha=1 / 128$. These bounds on $N_{E}$ and $N_{Q}$ are very loose; however, our criterion can be extended to every monomial of every $\beta$ function. We crucially observe that the size of $\lambda$ is not protected by any symmetry. Indeed, in the limit $\lambda \rightarrow 0$ there is no enhancement of the global symmetry. This also means that $\lambda$ is the most favorable coupling to get very large radiative corrections, so we expect $\left|\beta_{\lambda} / \lambda\right|<1$ to give important constraints. In particular, considering the various terms in (3.8), we derive the following bounds:

$$
\begin{aligned}
\frac{3}{16 \pi^{2}}|\lambda| & <1, \\
\frac{3}{2 \pi^{2}} N_{Q} y_{q}^{2} & <1, \\
\frac{1}{2 \pi^{2}} N_{E} y_{e}^{2} & <1, \\
\frac{9}{\pi^{2}} N_{Q} y_{q}^{4} & <|\lambda|, \\
\frac{3}{\pi^{2}} N_{E} y_{e}^{4} & <|\lambda| .
\end{aligned}
$$

The bound on $\lambda$ derived in this way is again very loose but we can find a maximum allowed value for this parameter imposing the condition of non-violation of perturbative unitary for the scattering of scalars $S S \rightarrow S S$. The analysis of the s-wave at tree-level gives $|\lambda|<16 \pi$. Making the conservative assumption $|\lambda| \leq 16 \pi$, from (3.14) and (3.15), we get:

$$
N_{Q} y_{q}^{4}<55.1, \quad N_{E} y_{e}^{4}<165 .
$$

It is possible to extract other bounds from $\left|\frac{\beta_{y_{q}}}{y_{q}}\right|<1$ and $\left|\frac{\beta_{y_{e}}}{y_{e}}\right|<1$ but it turns out that the constraints derived from $\left|\beta_{\lambda} / \lambda\right|<1$ are always stronger in any region of the parameter 
space. We are now ready to compare these bounds with the information on the model parameters coming from data on $S$. To this end, we can use the expression of section 3 of [77] adapted to the case of CP-odd interactions. The induced widths from fermion loops are given by

$$
\begin{aligned}
\Gamma(S \rightarrow g g) & =M \frac{\alpha_{3}^{2}}{8 \pi^{3}} N_{Q}^{2} y_{q}^{2} \tau_{Q}\left|\mathcal{P}\left(\tau_{Q}\right)\right|^{2}, \\
\Gamma(S \rightarrow \gamma \gamma) & =M \frac{\alpha^{2}}{16 \pi^{3}} Y^{4} N_{E}^{2} y_{e}^{2} \tau_{E}\left|\mathcal{P}\left(\tau_{E}\right)\right|^{2},
\end{aligned}
$$

where $\tau_{Q}=4 M_{Q}^{2} / M^{2}$ and $\tau_{E}=4 M_{E}^{2} / M^{2}$ and the loop function is defined as

$$
\mathcal{P}(\tau)=\arctan ^{2}(1 / \sqrt{\tau-1}) .
$$

In order to be conservative, we take the values of mediator masses close to their expected experimental exclusion limit. In particular, we take $M_{Q}=1 \mathrm{TeV}$ and $M_{E}=400 \mathrm{GeV}$. The decay widths normalized to the mass of the scalar are given by

$$
\frac{\Gamma(S \rightarrow g g)}{M}=5.7 \cdot 10^{-6} y_{q}^{2} N_{Q}^{2}, \quad \frac{\Gamma(S \rightarrow \gamma \gamma)}{M}=1.1 \cdot 10^{-7} Y^{4} y_{e}^{2} N_{E}^{2}
$$

and we neglected corrections of order $\left(M / 2 M_{Q}\right)^{2}$ and $\left(M / 2 M_{E}\right)^{2}$, respectively. Imposing the bounds on the product of $\Gamma_{\gamma \gamma} \Gamma_{g g}$, we obtain

$$
Y^{4} N_{E}^{2} N_{Q}^{2} y_{e}^{2} y_{q}^{2}=7.2 \cdot 10^{4}
$$

Extra constraints can be derived considering other phenomenological aspects. The non-observation of any significant excess in the di-photon invariant mass distribution at the $8 \mathrm{TeV}$ run at the LHC, suggests that the production cross section increases sizeably when varying the energy from $\sqrt{s}=8 \mathrm{TeV}$ to $\sqrt{s}=13 \mathrm{TeV}$. This fact favours production mechanisms of the scalar $S$ with large gain factor $r \equiv \sigma_{13 \mathrm{TeV}} / \sigma_{8} \mathrm{TeV}$. In our toy model, the scalar $S$ can be produced by gluon or photon fusion, and the respective gain factors are given by $r_{\gamma \gamma}=1.9$ and $r_{g g}=4.7$. Henceforth, to have a better fit of the $8 \mathrm{TeV}$ and $13 \mathrm{TeV}$ data, we impose in our model that the gluon production dominates over the photon one:

$$
C_{g g} \Gamma_{g g}>C_{\gamma \gamma} \Gamma_{\gamma \gamma}
$$

where $C_{g g}=2137$ and $C_{\gamma \gamma}=54$ are the partonic integrals as defined in [77]. In terms of our parameters we get

$$
y_{q}^{2} N_{Q}^{2}>4.9 \cdot 10^{-4} Y^{4} y_{e}^{2} N_{E}^{2} .
$$

An upper bound on $\Gamma_{g g}$ can be derived using results for searches for resonances decaying to di-jet final states [82]. From the analysis of [77] we infer that $\Gamma_{g g} / M<2 \cdot 10^{-3}$ and this gives:

$$
y_{q}^{2} N_{Q}^{2}<2.6 \cdot 10^{5} .
$$

We are now ready to collect all the information and to check in which region of the parameter space the model can be considered calculable according to our criterion (3.3). 
We obtain

$$
\begin{cases}N_{Q}<200 & \text { from }\left|\beta_{g_{s}} / g_{s}\right|<1, \\ N_{E} Y^{2}<1580 & \text { from }\left|\beta_{g^{\prime}} / g^{\prime}\right|<1, \\ N_{Q} y_{q}^{2}<6.6 & \text { from }\left|\beta_{\lambda} / \lambda\right|<1, \\ N_{E} y_{e}^{2}<19.7 & \text { from }\left|\beta_{\lambda} / \lambda\right|<1, \\ N_{Q} y_{q}^{4}<55.1 & \text { from }\left|\beta_{\lambda} / 16 \pi\right|<1, \\ N_{E} y_{e}^{4}<165 & \text { from }\left|\beta_{\lambda} / 16 \pi\right|<1, \\ Y^{2} N_{E} N_{Q} y_{e} y_{q}=268 & \sigma(p p \rightarrow S \rightarrow \gamma \gamma)=8 \mathrm{fb}, \\ Y^{2}\left|\frac{y_{e}}{y_{q}}\right| \frac{N_{E}}{N_{Q}}<45 & C_{g g} \Gamma_{g g}>C_{\gamma \gamma} \Gamma_{\gamma \gamma}, \\ \left|y_{q}\right| N_{Q}<513 & \Gamma_{g g} / M<2 \cdot 10^{-3},\end{cases}
$$

and will use the numerical analysis of this model to draw conclusions that are rather general. Moreover, we will comment on the range of applicability of our analysis and, finally, will provide the $\beta$ function for a generic gauge-Yukawa model with a single scalar field.

The analysis of the system of constraints in (3.25) suggests two scenarios where the model is calculable at the threshold of new physics states:

\section{Large hypercharge scenario.}

If the hypercharge of the new states is very large, then all of our constraints can be easily satisfied. The qualitative reason is again linked to the radiative correction of the self-interaction $\lambda$. Indeed, if we change the value of $Y$, all the constraints generated by $\left|\beta_{\lambda} / \lambda\right|<1$ remain unaffected, while the contribution to $\Gamma_{\gamma \gamma}$ gets a sufficient enhancement to explain the properties of the $750 \mathrm{GeV}$ resonance (including the large decay width). Notice that a change in the $\mathrm{SU}(3)_{C}$ color irreducible representation can be more dangerous because even if we benefit from an enhancement in $\Gamma_{g g}$ due to larger group invariant $C_{r}$, the enlargement of the dimensionality of the irreducible representation gives a larger correction to the quartic $\lambda$.

\section{Large multiplicity scenario.}

If we want to avoid very large and exotic electric charges, then we are forced to consider a sizable number of states. In the following we will consider $Y=1$ for concreteness to present the argument. First, just considering the seventh row of (3.25), it seems possible to address the excess by assuming Yukawa couplings of $y_{e} \sim y_{q} \sim 1$ with $N_{E} \sim N_{Q} \sim 16$ or even $y_{e} \sim y_{q} \sim \mathcal{O}(5)$ with only $N_{E} \sim N_{Q} \sim 3$. However, taking into account in particular the third and fourth rows, such solutions are excluded. So we make the crucial observation that the running of the scalar quartic renders scenarios trying to address the signal with sizable Yukawa couplings and a modest number of new states uncalculable. A very large number of new states seems unavoidable. Quantitatively, from the third, fourth, and seventh rows in (3.25) above, we obtain the interesting bounds

$$
N_{E} N_{Q}>550,
$$




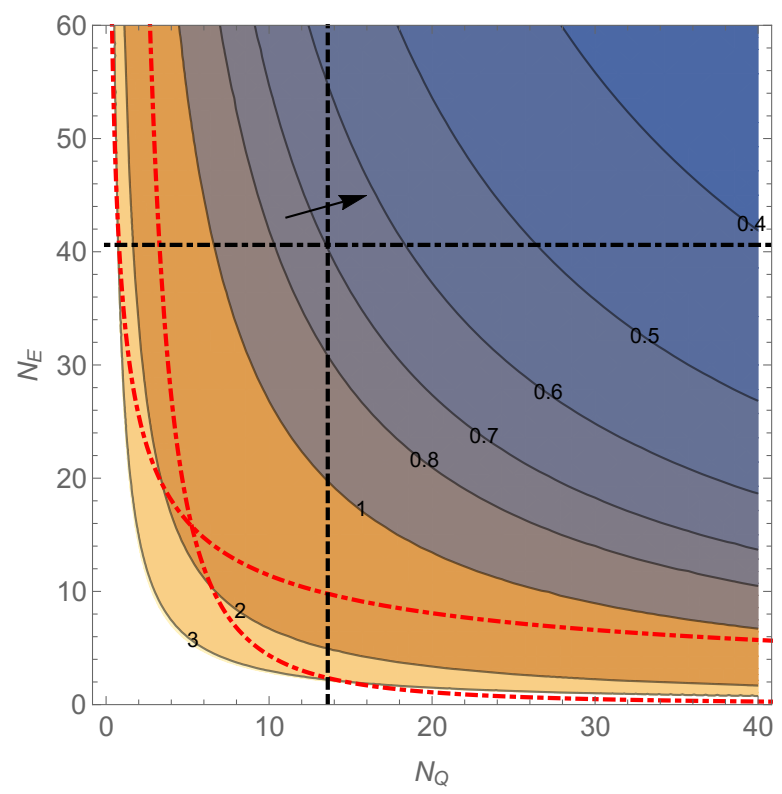

Figure 1. Contours of constant Yukawa couplings $y_{e}=y_{q}$ in the $N_{E}$ vs. $N_{Q}$ plane for parameter points reproducing the di-photon resonance. The bounds from the running of the scalar quartic from the third and forth rows of (3.25) are depicted by black dashed-dotted lines, while bounds from the fifth and sixth rows are depicted by red dashed-dotted lines. The arrow denotes the region of parameters were all constraints are satisfied. See text for details.

and

$$
y_{e} y_{q}<0.49
$$

Finally, we present a quantitative overview on the number of required new fermions and its interplay with the Yukawa couplings of the new scalar in figure 1. Here, we show contours of constant Yukawa couplings $y_{e}=y_{q}$, which we assume equal for simplicity, in the $N_{E}$ vs. $N_{Q}$ plane, where we require the seventh row of (3.25) to be fulfilled. The allowed region is obtained for

$$
41<N_{E}<1580, \quad 14<N_{Q}<200 .
$$

Although the model can be considered calculable according to (3.3), the vary large number of states generates a Landau pole very close to the $\mathrm{TeV}$ scale.

The considerations made for our toy model are rather general and we believe that the problem of calculability is an issue in a large part of the proposed weakly coupled solutions of the $750 \mathrm{GeV}$ puzzle. For this reason, we suggest applying our simple strategy in order to check if a model is calculable and predictions can be trusted. We summarize here the main considerations to perform this analysis:

- Our criterion is based on a comparison between tree-level effects and one-loop corrections. The comparison is done confronting the tree-level value of the couplings with 
their $\beta$ function:

$$
\left|\frac{\beta_{y_{i}}^{(1)}(\{y\})}{y_{i}}\right|<1 \quad \text { for every coupling } y_{i}
$$

- One should write the complete sets of beta functions for a given model, these can be found for example in [78-81].

- If we don't allow cancellations of the various terms in the $\beta$ functions, our condition can be applied to every monomial .

- This will give a large number of constraints on the parameters space, and one has to check if this region allows for an explanation of the $750 \mathrm{GeV}$ resonance.

- We also want to remark that a quartic coupling for the new scalar state is always allowed in a renormalizable model. Moreover, this term is not protected by any symmetry, so it is the ideal place to look for large radiative corrections. ${ }^{4}$

- Perturbative unitarity bounds can be used to set upper bounds on the couplings providing further constrains; for example, those coming from $2 \rightarrow 2$ scattering of the new scalar are particularly important to bound the quartic self-coupling.

We conclude by noticing that several explanations of the $750 \mathrm{GeV}$ resonance consider just the SM augmented by a single real scalar and a series of fermions. For this case, we provide simple and compact formula for the $\beta$ functions based on the results in [81]. We consider the following lagrangian for a simple gauge group

$$
\begin{aligned}
\mathcal{L}= & -\frac{1}{4} F^{A \mu \nu} F_{\mu \nu}^{A}+\frac{1}{2} \partial^{\mu} S \partial_{\mu} S+i \psi_{i}^{\dagger} \sigma^{\mu} D_{\mu} \psi_{i} \\
& -\frac{1}{2}\left(\underline{Y}_{i j} \psi_{i} \psi_{j} S+\text { h.c. }\right)-\frac{1}{4 !} S^{4} .
\end{aligned}
$$

Lorentz contractions are implicit and we consider only marginal operators. The matrix $\underline{Y}$ is such that it extends over all the indices of the fields including the gauge ones. For example, for a pair of vector-like QCD triplets $Q$ and $Q^{c}, \underline{Y}$ is symmetric matrix of order 6. We also need the following definitions:

$$
\begin{aligned}
S_{2}(F) \delta^{A B} & =\operatorname{Tr}\left(t^{A} t^{B}\right), \\
C_{2}(G) \delta^{A B} & =f^{A C D} f^{B C D}, \\
C_{2}^{a b}(F) & =t_{a c}^{A} t_{c b}^{A},
\end{aligned}
$$

where $t^{A}$ are the generators of the gauge group acting on the fermion fields and $f^{A B C}$ are the structure constant of the simple group. The capital letter $F$ refers to the (in general reducible) representation of the fermions under $G$ denote the adjoint irreducible

\footnotetext{
${ }^{4}$ With few exceptions: $D$-terms in SUSY extensions, where quartic scalars are protected by gauge symmetry and the goldstone boson case, where the protection is given by the shift symmetry.
} 
representation of the gauge group. The $\beta$ functions are given by:

$$
\begin{aligned}
& \beta_{g}=-\frac{g^{3}}{(4 \pi)^{2}}\left(\frac{11}{3} C_{2}(G)-\frac{4}{3} \kappa S_{2}(F)\right), \\
& \beta_{\lambda}=\frac{1}{16 \pi^{2}}\left(3 \lambda^{2}+8 \kappa \operatorname{Tr}\left(\underline{Y}^{\dagger} \underline{Y}\right) \lambda-48 \kappa \operatorname{Tr}\left(\left(\underline{Y}^{\dagger} \underline{Y}\right)^{2}\right)\right), \\
& \beta_{\underline{Y}}=\frac{1}{2} \underline{Y}^{\dagger} \underline{Y}^{2}+\frac{5}{2} \underline{Y} \underline{Y}^{\dagger} \underline{Y}+2 \kappa \underline{Y} \operatorname{Tr}\left(\underline{Y} \underline{Y}^{\dagger}\right),-3 g^{2}\left\{C_{2}(F), \underline{Y}\right\}
\end{aligned}
$$

where the parameter $\kappa$ is set to $1 / 2$ for the case of two-component fermions. An extension to non-simple groups $G_{1} \times \cdots \times G_{n}$ requires the substitutions:

$$
\begin{aligned}
g^{3} C_{2}(G) & \rightarrow \sum_{k} g_{k}^{3} C_{2}\left(G_{k}\right), \\
g^{3} S_{2}(F) & \rightarrow \sum_{k} g_{k}^{3} S_{2}^{k}(F), \\
g^{2} C_{2}(F) & \rightarrow \sum_{k} g_{k}^{2} C_{2}^{k}(F),
\end{aligned}
$$

where $g_{k}$ is the gauge coupling of the group $G_{k}$. As we argued in this section, we expect the most important constraints to came from the radiative effects on $\lambda$. In particular we get

$$
\begin{aligned}
& \left|\frac{\beta_{\lambda}}{\lambda}\right|<1 \Longrightarrow \operatorname{Tr}\left(\underline{Y}^{\dagger} \underline{Y}\right)<4 \pi^{2} \approx 39, \\
& \left|\frac{\beta_{\lambda}}{16 \pi}\right|<1 \Longrightarrow \operatorname{Tr}\left(\left(\underline{Y}^{\dagger} \underline{Y}\right)^{2}\right)<\frac{2 \pi^{2}}{3} 16 \pi \approx 330 .
\end{aligned}
$$

\section{Constraints on couplings to SM fermions}

As discussed in section 2, we can parametrize the relevant $S$ couplings to SM fermions after electroweak (EW) symmetry breaking — in the mass basis — as

$$
\mathcal{L}_{Y} \ni-\sum_{\ell_{i}, \ell_{j}=e, \mu, \tau} Y_{\ell_{i} \ell_{j}} S \bar{\ell}_{i} P_{L} \ell_{j}-\sum_{d, d_{j}=d, s, b} Y_{d_{i} d_{j}} S \bar{d}_{i} P_{L} d_{j}-\sum_{u_{i}, u_{j}=u, c, t} Y_{u_{i} u_{j}} S \bar{u}_{i} P_{L} u_{j}+\text { h.c. . }
$$

In the following we also fix $\Lambda_{i}=1 \mathrm{TeV}$ in (2.3). All our results however can be easily rescaled to any other values. Considering effects well below the scale of $M$, we can also safely use (2.3) to parametrize the constraints coming from the observations of $S$ at the LHC. The summary of our derived constraints on $Y_{i j}$ and consequently $S$ decay products with these assumptions is given in table 1. The details of their derivation are however presented in the following two subsections.

\subsection{Dipole moments}

Important information and constraints on the flavor and CP structure of $S$ couplings to SM fermions can be extracted from existing low energy measurements and searches. In particular, focusing on the flavor diagonal couplings, the measured MDMs of SM charged leptons, the constraints on their EDMs, as well as constraints on nuclear EDMs provide 


\begin{tabular}{|c|c|c|}
\hline Bound on $Y_{f, f^{\prime}}$ & Observable & $\Gamma\left(S \rightarrow f f^{\prime}\right) / M$ \\
\hline$\left|\operatorname{Im}\left(Y_{e e}\right)\right| \lesssim 1 \times 10^{-7}$ & $d_{e}$ & $\lesssim 6 \times 10^{-16}$ \\
\hline$\left|\operatorname{Im}\left(Y_{d d}\right)\right| \lesssim 3 \times 10^{-4}$ & $d_{N}, d_{\mathrm{Hg}}$ & $\lesssim 1 \times 10^{-8}$ \\
$\left|\operatorname{Im}\left(Y_{u u}\right)\right| \lesssim 5 \times 10^{-4}$ & $d_{N}, d_{\mathrm{Hg}}$ & $\lesssim 3 \times 10^{-8}$ \\
\hline$\left|Y_{e \mu}\right|,\left|Y_{\mu e}\right| \lesssim 2 \times 10^{-5}$ & $\mathcal{B}(\mu \rightarrow e \gamma)$ & $\lesssim 1 \times 10^{-11}$ \\
$\left|Y_{e \tau}\right|,\left|Y_{\tau e}\right| \lesssim 0.08$ & $\mathcal{B}(\tau \rightarrow e \gamma)$ & $\lesssim 3 \times 10^{-4}$ \\
$\left|Y_{\mu \tau}\right|,\left|Y_{\tau \mu}\right| \lesssim 0.09$ & $\mathcal{B}(\tau \rightarrow \mu \gamma)$ & $\lesssim 3 \times 10^{-4}$ \\
\hline$\sqrt{\operatorname{Re}\left[\left(Y_{s d}\right)^{2}\right]}, \sqrt{\operatorname{Re}\left[\left(Y_{d s}\right)^{2}\right]}<1.3 \times 10^{-4}$ & $\Delta m_{K}$ & $<2.0 \times 10^{-9}$ \\
$\sqrt{\operatorname{Im}\left[\left(Y_{s d}\right)^{2}\right]}, \sqrt{\operatorname{Im}\left[\left(Y_{d s}\right)^{2}\right]}<3.4 \times 10^{-6}$ & $\epsilon_{K}$ & $<1.4 \times 10^{-12}$ \\
$\sqrt{\operatorname{Re}\left[\left(Y_{c u}\right)^{2}\right]}, \sqrt{\operatorname{Re}\left[\left(Y_{u c}\right)^{2}\right]}<3.3 \times 10^{-4}$ & $x_{D}$ & $<1.3 \times 10^{-8}$ \\
$\sqrt{\operatorname{Im}\left[\left(Y_{c u}\right)^{2}\right]}, \sqrt{\operatorname{Im}\left[\left(Y_{u c}\right)^{2}\right]}<4.0 \times 10^{-5}$ & $(q / p)_{D}, \phi_{D}$ & $<1.9 \times 10^{-10}$ \\
$\sqrt{\operatorname{Re}\left[\left(Y_{b d}\right)^{2}\right]}, \sqrt{\operatorname{Re}\left[\left(Y_{b d}\right)^{2}\right]}<4.1 \times 10^{-4}$ & $\Delta m_{d}$ & $<2.0 \times 10^{-8}$ \\
$\sqrt{\operatorname{Im}\left[\left(Y_{b d}\right)^{2}\right]}, \sqrt{\operatorname{Im}\left[\left(Y_{b d}\right)^{2}\right]}<2.3 \times 10^{-4}$ & $\sin 2 \beta$ & $<6.3 \times 10^{-9}$ \\
$\left|\left(Y_{b s}\right)\right|,\left|\left(Y_{s b}\right)\right|<1.7 \times 10^{-3}$ & $\Delta m_{s}$ & $<3.4 \times 10^{-7}$ \\
\hline
\end{tabular}

Table 1. Constraints on $S$ couplings to SM fermions (first column) derived from low energy precision observables (second column). Results in first seven rows assume CP-even $S\left(\tilde{\epsilon}_{g}=\tilde{\epsilon}_{\gamma}=0\right)$, $\left|\epsilon_{\gamma}\right|>0.05$ and $\left|\epsilon_{g}\right|>1 \times 10^{-3}$ in order to reproduce the observed di-photon signal from gluon fusion. The case of CP-odd $S$ can be obtained via replacement of $\operatorname{Im} \rightarrow$ Re. Only a single $Y_{i j}$ is assumed to be non vanishing in each column. The associated limits on the $S$ decay rates to the corresponding flavored final states are presented in the third column.

very stringent probes. In order to simplify the notation in this section we will explicitly split each flavor diagonal $S$ Yukawa coupling into its real and imaginary parts as

$$
Y_{i i} \equiv Y_{i}^{\prime}+i \tilde{Y}_{i}^{\prime}
$$

where $Y_{i}^{\prime}$ and $\tilde{Y}_{i}^{\prime}$ are defined as real. We start by defining the NP induced SM lepton EDMs and shifts to MDMs via

$$
\mathcal{L}_{\text {dipole }}^{\ell}=-\sum_{\ell} \frac{1}{4 m_{\ell}} \bar{\ell} \sigma^{\mu \nu}\left(e \Delta a_{\ell}+2 i m_{\ell} d_{\ell} \gamma_{5}\right) \ell F_{\mu \nu} .
$$

We first analyze the constraints (as well as possible NP hints) coming from the lepton MDM measurements. The current experimental situation can be summarized as follows [83-85]

$$
\begin{aligned}
\left|\Delta a_{e}\right| & <8 \times 10^{-12} \\
\Delta a_{\mu} & =(2.92 \pm 0.86) \times 10^{-9} \\
-0.052 & <\Delta a_{\tau}<0.013
\end{aligned}
$$

where in the second row we have added the various experimental and theoretical errors in quadrature and took the central value as quoted in the PDG [85]. Perhaps the most 
interesting part here is the long standing anomaly in $a_{\mu}$. If explained due to the same new particles, which are responsible for the $\gamma \gamma$ excess at $750 \mathrm{GeV}$, it can provide unambiguous information on the couplings of the new sector to muons and further correlate $a_{\mu}$ with existing constraints on the $S$ decays to $\mu^{+} \mu^{-}$.

The dominant contribution to the muon MDM comes from the so called Barr-Zee type diagram in figure 2, very similar to the structure shown in [86-90] in the context of the Two Higgs doublet model (THDM). ${ }^{5}$ There are however several important differences between the perturbative THDM scenarios and the required properties of $S$. First, as discussed in the previous section the substantial coupling of $S$ to the di-photons is likely to be explained within a non-perturbative UV completion. In accordance with this and in order to simplify the expressions, we will also completely neglect the SM fermions running in the loop and concentrate on the UV contributions in (2.3). Second, the likely nonperturbativity of the UV completion poses a clear challenge to the explicit calculation of the Barr-Zee diagrams when the effective couplings between $S$ and photons emerges from strong dynamics. To emeliorate this problem, we consider in our analysis only the universal pieces, logarithmically sensitive to the UV dynamics which scale as $\log \left(\Lambda_{i}\right) / \Lambda_{i}$, but neglect all the finite terms which scale as $1 / \Lambda_{i}$, because the latter are model dependent.

Before we go into the details of the calculation, it is instructive to estimate the effect from considerations of dimensional analysis. Being a logarithmically-divergent contribution to a dimension-five operator, it is expected to scale as

$$
\Delta a_{\ell} \sim \epsilon_{\gamma} \frac{m_{\ell}}{\Lambda_{\gamma}} \frac{\alpha Y_{\ell}^{\prime}}{4 \pi} \log \frac{\Lambda_{\gamma}^{2}}{M^{2}}+\text { finite }
$$

where $\Lambda_{\gamma}$ is the mass scale of heavy (strongly coupled) states generating $S \rightarrow \gamma \gamma$. This structure immediately suggests that accommodating the reported value of the $a_{\mu}$ anomaly (4.5) requires $Y_{\mu}^{\prime} \sim 0.1$, in conflict with the non-observation of $S$ in the $\mu^{+} \mu^{-}$ channel. We will now show that this naive estimation is in fact fairly close to the result of the explicit calculation.

In our estimate we assume that the structure of the $S \gamma \gamma$ vertex is identical to one gets from loops of heavy fermions (see [91] for the details), namely

$$
i \Gamma^{\mu \nu} \propto i \Lambda\left(g^{\mu \nu} k \cdot q-k^{\mu} q^{\nu}\right) \int_{0}^{1} d x \frac{2 x(1-x)-1}{k^{2}(1-x)-\Lambda^{2}},
$$

where $q^{\mu}$ stands for the momentum of the external photon, $k^{\mu}$ denotes the momentum running in the loop, and $\Lambda$ is the mass of the heavy fermion running in the loop. Of course, at the end of the day we will take the limit $\Lambda \rightarrow \infty$ to recover the effective dimension-5 operator. The first part of this expression is completely generic and relies on no model

\footnotetext{
${ }^{5}$ We note in passing that while focusing here on an EW singlet $S$, our results are expected to apply also to large parts of the parameter space of models where $S$ is the neutral component of a larger multiplet. The main difference is due to the fact that in the singlet case there is only as single neutral state which gives a new contribution to the MDM. In a THDM on the other hand one in general needs to include both $\mathrm{CP}$-even and CP-odd states. Moreover, these contributions are suppressed only by $\log M$ and therefore heavier states are potentially as important as of the lightest one. Hereafter we assume only one state in our analysis, but extension to two or more states is possible along these lines.
} 


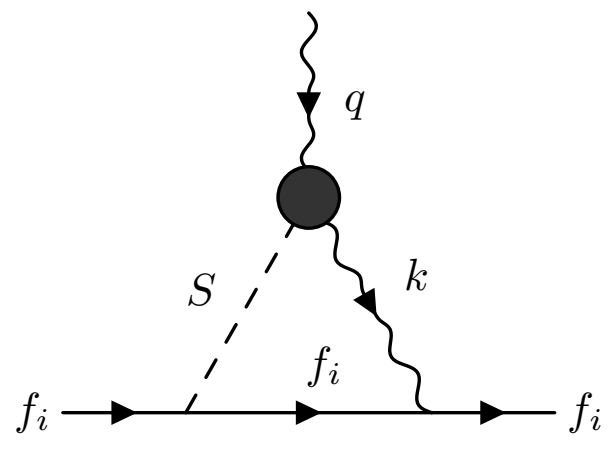

(a)

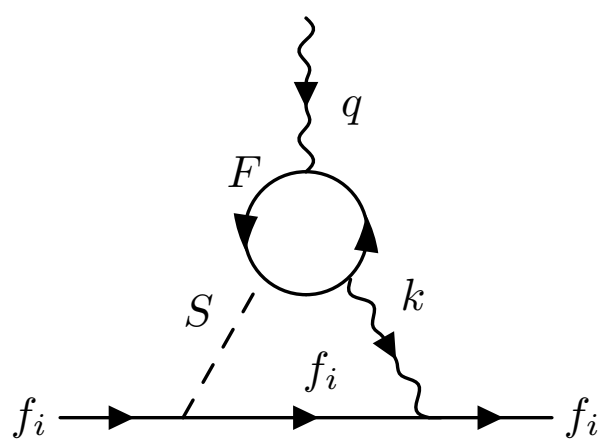

(b)

Figure 2. Barr-Zee diagrams which contribute to the EDMs and MDMs or the SM fermions. The diagram on the RH side shows the full perturbative two-loop process, while in the diagram on the LH-side we replace the $S \gamma \gamma$ interaction by an effective vertex.

assumptions. It is only the integral which depends on the structure generated by integrating out heavy fermions. However, we expect these details of the vertex structure to be relevant only for the finite terms, and not for the leading logarithm that we are interested in.

From here it is easy to write down the expression for the new contribution to the lepton MDM, closely following [91]:

$$
\Delta a_{\ell} \simeq-2 \epsilon_{\gamma} \frac{m_{\ell}}{\Lambda_{\gamma}} \frac{\alpha Y_{\ell}^{\prime}}{\pi} \log \left(\frac{\Lambda_{\gamma}}{M}\right)-2 \tilde{\epsilon}_{\gamma} \frac{m_{\ell}}{\Lambda_{\gamma}} \frac{\alpha \tilde{Y}_{\ell}^{\prime}}{\pi} \log \left(\frac{\Lambda_{\gamma}}{M}\right)
$$

where we have explicitly integrated out the heavy fermions. ${ }^{6}$ In the case of $\ell=\mu$, a comparison with the reported value of $a_{\mu}$ yields at the $2 \sigma$ level

$$
8.5 \times 10^{-3} \lesssim\left|\epsilon_{\gamma} Y_{\mu}^{\prime}\right| \lesssim 3.3 \times 10^{-2} \quad \text { for } \Lambda_{\gamma}=1 \mathrm{TeV}
$$

Of course similar values apply for the combination $\tilde{\epsilon}_{\gamma} \tilde{Y}_{\mu}^{\prime}$ if the $S$ is (predominantly) a pseudo-scalar. These numbers should be taken with a grain of salt because of our approximation of the effects of the dimension-five operator by a simple logarithm. In particular, given that the expected separation of $M$ and $\Lambda_{\gamma}$ scales is expected to be moderate at most, the model dependent finite contributions could be comparable in specific UV-models.

Given that $S \rightarrow \mu^{+} \mu^{-}$non-observation implies $\left|Y_{\mu}^{\prime}\right| \lesssim 0.06$, we see that interpreting $\Delta a_{\mu}$ as a signal is marginally consistent with $\Lambda_{\gamma}$ being close to TeV. More importantly, the preferred range of $\left|Y_{\mu}^{\prime}\right|$ will be probed in the very near future at the LHC13. Note also, that in order to interpret $\Delta a_{\mu}$ as a signal one is forced to assume $\epsilon_{\gamma} \gtrsim 0.1$. This assumption is in tension with non observation of $S$ in the di-photon final state at LHC8 and suggests that the theory might have non-perturbative UV-completion [77]. If instead interpreted

\footnotetext{
${ }^{6}$ From integrating out the heavy fermions loop we have $\epsilon_{\gamma}=\frac{N_{f} Q_{f}^{2} Y_{f}}{12 \pi^{2}}$ and $\tilde{\epsilon}_{\gamma}=\frac{N_{f} Q_{f}^{2} \tilde{Y}_{f}}{8 \pi^{2}}$. One can also match $\epsilon_{g}=\frac{N_{f} I_{f} Y_{f}}{12 \pi^{2}}$ and $\tilde{\epsilon}_{g}=\frac{N_{f} I_{f} \tilde{Y}_{f}}{8 \pi^{2}}$. In these expressions $I_{f}=1 / 2$ for the fundamentals of the SU(3) and $I_{f}=3$ for the adjoint.
} 
as a constraint, $a_{\mu}$ implies no bound which would be stronger than existing direct LHC searches in the $\mu^{+} \mu^{-}$channel.

When we apply these considerations to $\epsilon_{\gamma} Y_{\tau}^{\prime}$ and $\tilde{\epsilon}_{\gamma} \tilde{Y}_{\tau}^{\prime}$, we get no meaningful bound, while bounds on the couplings of the new scalar to the electrons are marginal

$$
\left|\epsilon_{\gamma} Y_{e}^{\prime}\right|,\left|\tilde{\epsilon}_{\gamma} \tilde{Y}_{e}^{\prime}\right| \lesssim 1.17 \times 10^{-2} \quad \text { for } \Lambda_{\gamma}=1 \mathrm{TeV},
$$

which is comparable to the existing direct LHC constraints.

Turning to the leptonic EDMs, they constrain the CP-violating products of $S$ couplings to the charged SM leptons and photons $\left(\epsilon_{\gamma} \tilde{Y}_{\ell}^{\prime}\right.$ and/or $\left.\tilde{\epsilon}_{\gamma} Y_{\ell}^{\prime}\right)$. The current experimental bounds read

$$
\begin{array}{r}
\left|d_{e}\right|<8.7 \times 10^{-29} \mathrm{e} \mathrm{cm}, \\
d_{\mu}=(-0.1 \pm 0.9) \times 10^{-19} \text { e cm } \\
\left(-0.25<d_{\tau}<0.008\right) \times 10^{-16} \mathrm{e} \mathrm{cm} .
\end{array}
$$

We estimate the contributions of $S$ using the analogous expressions for the corresponding Barr-Zee diagrams, which were obtained for the hypothetic CP-violating Higgs couplings to the third generation fermions in [92].

As in the MDM case, effectively integrating out the heavy fermions and keeping only the leading logarithmic pieces we get the following expression for the new contribution to the lepton EDMs:

$$
\frac{d_{\ell}}{e} \simeq-\epsilon_{\gamma} \frac{\alpha}{\pi} \frac{\tilde{Y}_{\ell}^{\prime}}{\Lambda_{\gamma}} \log \left(\frac{\Lambda_{\gamma}}{M}\right)
$$

Of course, similar contributions proportional to the $\tilde{\epsilon}_{\gamma} Y_{\ell}^{\prime}$ will be formed. Evidently, no meaningful constraints exist on the CP-violating couplings either of the tau lepton or of the muon. However, the constraint on the $\mathrm{CP}$-violating coupling to the electron is stringent

$$
\left|\tilde{\epsilon}_{\gamma} Y_{e}^{\prime}\right|,\left|\epsilon_{\gamma} \tilde{Y}_{e}^{\prime}\right|<6.2 \times 10^{-9}
$$

depending whether the contribution to the EDM is positive or negative (a priori, we do not know the sign of the products $\left.\epsilon_{\gamma} \tilde{Y}_{e}^{\prime}, \tilde{\epsilon}_{\gamma} Y_{e}^{\prime}\right)$.

Finally we analyze the CP-violating couplings of $S$ to the SM quarks. These are bounded by the measurements of nuclear EDMs, the most stringent constraints currently coming from the EDMs of the neutron and the nucleus of mercury. A priori, there are several different contributions to nuclear EDMs. Here we focus on quark EDMs, chromo EDMs (CEDMS), CP-violating nucleon-pion interactions and the contribution from the three-gluon Weinberg operator. In our analysis we follow the procedure of [92, 93], defining the effective operators contributing the (C)EDMs as

$$
\begin{aligned}
\mathcal{L}_{C / P}^{q}= & \sum_{q}\left[i \frac{c_{q}}{2} e Q_{i} \bar{q} \sigma^{\mu \nu} \gamma_{5} q F_{\mu \nu}+i \frac{c_{q}^{c}}{2} g_{s} q \sigma^{\mu \nu} T^{a} \gamma_{5} q G_{\mu \nu}^{a}+i C_{q}(\bar{q} q)\left(\bar{q} \gamma_{5} q\right)\right] \\
& +\frac{c_{W}}{6} g_{s} f^{a b c} G_{\mu \rho}^{a} G_{\nu}^{b \rho} G_{\lambda \sigma}^{c} \epsilon^{\mu \nu \lambda \sigma} .
\end{aligned}
$$


Analogously to the case of leptonic EDMs, the Wilson coefficients of the EDM and CEDM operators at the matching scale (at the leading logarithmic order) are

$$
\begin{aligned}
& c_{q} \simeq \epsilon_{\gamma} \frac{\alpha}{\pi} \frac{\tilde{Y}_{q}^{\prime}}{\Lambda_{\gamma}} \log \left(\frac{\Lambda_{\gamma}}{M}\right), \\
& c_{q}^{c} \simeq \epsilon_{g} \frac{\alpha_{s}}{\pi} \frac{\tilde{Y}_{q}^{\prime}}{\Lambda_{\gamma}} \log \left(\frac{\Lambda_{\gamma}}{M}\right),
\end{aligned}
$$

and similarly for $\tilde{\epsilon}_{\gamma} Y_{q}^{\prime}, \tilde{\epsilon}_{g} Y_{q}^{\prime}$ combinations. We further run these operators to the hadronic scale $\mu_{H} \sim 1 \mathrm{GeV}$ using the known QCD expressions [94, 95], including finite shifts of the Weinberg operator at heavy quark thresholds $[96,97]$ and match them onto the neutron and mercury EDMs (cf. [98]). Using the recently updated values of the relevant hadronic matrix elements $[99,100]$ we obtain

$$
\begin{gathered}
d_{n}=0.774(66) e Q_{d} c_{d}-0.233(28) e Q_{u} c_{u}+1.1(5) e\left(c_{d}^{c}+0.5 c_{u}^{c}\right)+22(10) \times 10^{-3}{\mathrm{GeV} e c_{W},}, \\
d_{\mathrm{Hg}}=7(4) \times 10^{-3} e\left(c_{u}^{c}-c_{d}^{c}\right)+1.4(7) \times 10^{-5} \mathrm{GeV}^{2} e\left(\frac{C_{u}}{m_{u}}-0.5 \frac{C_{d}}{m_{d}}\right) .
\end{gathered}
$$

in terms of the effective Wilson coefficients evaluated at the hadronic scale. Interestingly the nuclear EDM bounds (at $90 \%$ C.L.) of

$$
\left|d_{n}\right|<2.9 \times 10^{-26} \text { e cm, }\left|d_{\mathrm{Hg}}\right|<3.1 \times 10^{-29} \text { e } \mathrm{cm},
$$

impose meaningful bounds on the CP-violating couplings. ${ }^{7}$ In particular, conservatively taking the lower one-sigma values for all hadronic matrix elements, the neutron EDM yields

$$
\begin{aligned}
\left|\tilde{Y}_{d}^{\prime}\left[(69 \pm 28) \epsilon_{g}-\epsilon_{\gamma}\right]\right| & <1.6 \times 10^{-5}, \\
\left|\tilde{Y}_{u}^{\prime}\left[(60 \pm 24) \epsilon_{g}-\epsilon_{\gamma}\right]\right| & <2.6 \times 10^{-5}, \\
\left|\tilde{Y}_{c}^{\prime} \epsilon_{g}\right| & <9.0 \times 10^{-4}, \\
\left|\tilde{Y}_{b}^{\prime} \epsilon_{g}\right| & <9.6 \times 10^{-3}, \\
\left|\tilde{Y}_{t}^{\prime} \epsilon_{g}\right| & <1.8,
\end{aligned}
$$

while the mercury EDM gives

$$
\begin{aligned}
\left|\tilde{Y}_{d}^{\prime} \epsilon_{g}\right| & <8.7 \times 10^{-4} \\
\left|\tilde{Y}_{u}^{\prime} \epsilon_{g}\right| & <8.7 \times 10^{-4} .
\end{aligned}
$$

In addition, the mercury EDM is also sensitive to $\mathrm{CP}$ violating four-fermion interactions of 1 ight quarks [98], in particular tree level $S$ exchange yields

$$
C_{q}=\frac{Y_{q}^{\prime} \tilde{Y}_{q}}{M^{2}}
$$

\footnotetext{
${ }^{7}$ In our analysis we do not include the bound on the CP-violating coupling to the strange quark. The reason is that the neutron EDM dependence on this coupling is highly uncertain and consistent with zero [99-101].
} 
at the matching scale $M$. The QCD evolution to the hadronic scale [95] reduces $C_{q}$ by $C_{q}\left(\mu_{H}\right) \simeq 0.2 C_{q}(M)$ leading to the bounds

$$
\begin{aligned}
&\left|\tilde{Y}_{d}^{\prime} Y_{d}^{\prime}\right| \lesssim 8.9 \times 10^{-3}, \\
&\left|\tilde{Y}_{u}^{\prime} Y_{u}^{\prime}\right| \lesssim 2.0 \times 10^{-3} .
\end{aligned}
$$

Finally, similar effects due to heavy quarks can be relevant though their contributions to the Weinberg operator. The two loop contribution due to a massive quark $q$ can be factorized in terms of the mixing of $C_{q}$ into $\tilde{c}_{q}$ followed by a threshold shift of $c_{W}$ at the scale $m_{q}$. This yields [95]

$$
c_{W} \simeq \frac{4 \alpha_{s}}{(4 \pi)^{3}} \frac{Y_{q}^{\prime} \tilde{Y}_{q}^{\prime}}{M^{2}}\left[\log \left(\frac{M}{m_{q}}\right)-\frac{3}{4}\right],
$$

in agreement with an explicit evaluation of the two-loop result $[92,102]$. In this case the bound coming from the neutron EDM is much stronger and yields

$$
\begin{aligned}
&\left|\tilde{Y}_{c}^{\prime} Y_{c}^{\prime}\right| \lesssim 1.0 \times 10^{-2} \\
&\left|\tilde{Y}_{b}^{\prime} Y_{b}^{\prime}\right| \lesssim 4.3 \times 10^{-2} \\
&\left|\tilde{Y}_{t}^{\prime} Y_{t}^{\prime}\right| \lesssim 1.3 .
\end{aligned}
$$

\subsection{Flavor observables}

Next we derive constraints on $Y_{i \neq j}$ and consequently flavored $S$ decay products in table 1 . As before we restrict our discussion to the inclusion of a single non-vanishing $Y_{i j}$ at a time. Here the leading effects can appear already at the tree level. In particular, off-diagonal couplings to quarks will induce neutral meson oscillations through tree-level $S$ exchange

$$
\mathcal{L}_{\Delta F=2}=\sum_{i \neq j} \frac{Y_{d_{i} d_{j}}^{2}}{2 M^{2}}\left(\bar{d}_{i} P_{L} d_{j}\right)^{2}+\sum_{i \neq j} \frac{Y_{u_{i} u_{j}}^{2}}{2 M^{2}}\left(\bar{u}_{i} P_{L} u_{j}\right)^{2}+\text { h.c. } .
$$

The resulting constraints can be derived following the results of [103, 104]. The values shown in table 1 have been obtained taking into account the recent Lattice QCD updates [105-107]. We note that in case $S$ is a part of a larger $\mathrm{SU}(2)_{L}$ multiplet there can be additional effects coming from the associated charged scalars. Formally at least, these will be loop suppressed compared to contributions in (4.37). In practice however the relative size of both effects depends on the detailed flavor composition of the model.

The second class of effects is very similar to the contributions of flavor diagonal $Y_{i i}$ to the fermionic dipole moments discussed in the previous subsection. Namely, the simultaneous presence of $Y_{i j}$ and $\epsilon_{\gamma, g}$ generates radiative FCNC operators at the one-loop order. We define the relevant flavor changing dipole operators through

$$
\mathcal{L}_{f_{1}, f_{2}}=c_{L f_{1} f_{2} \gamma} Q_{L f_{1} f_{2} \gamma}+(L \leftrightarrow R)+(\gamma \leftrightarrow g),
$$

where

$$
Q_{L f_{1} f_{2} \gamma}=\frac{e}{8 \pi^{2}} \bar{f}_{2}(\sigma \cdot F) P_{L} f_{1}, \quad Q_{L f_{1} f_{2} g}=\frac{g_{s}}{8 \pi^{2}} \bar{f}_{2} T^{a}\left(\sigma \cdot G^{a}\right) P_{L} f_{1}
$$


and analogous for $Q_{R f_{1} f_{2} \gamma}$ and $Q_{R f_{1} f_{2} g}$. Using the results of existing calculations done in the context of renormalizable models $[104,108,109]$ we derive

$$
\begin{aligned}
& c_{L f_{1} f_{2} \gamma} \simeq 4 \pi \alpha \epsilon_{\gamma} Q_{f_{1}} \frac{Y_{f_{2} f_{1}}}{\Lambda_{\gamma}} \log \left(\frac{\Lambda_{\gamma}}{M}\right), \\
& c_{L f_{1} f_{2} g} \simeq 4 \pi \alpha_{s} \epsilon_{g} \frac{Y_{f_{2} f_{1}}}{\Lambda_{g}} \log \left(\frac{\Lambda_{g}}{M}\right),
\end{aligned}
$$

where we have again only kept the logarithmically enhanced contributions, and where $c_{R f_{1} f_{2} \gamma}=c_{L f_{2} f_{1} \gamma}^{*}, c_{R f_{1} f_{2} g}=c_{L f_{2} f_{1} g}^{*}$. Finally, contributions of $\tilde{\epsilon}_{\gamma}$ and $\tilde{\epsilon}_{g}$ can obtained directly from the above expressions via the replacements $\gamma \rightarrow \tilde{\gamma}, g \rightarrow \tilde{g}$ and $Y_{f_{2} f_{1}} \rightarrow Y_{f_{1} f_{2}}^{*}$. The partonic radiative rates are then given by

$$
\begin{aligned}
\Gamma\left(f_{1} \rightarrow f_{2} \gamma\right) & =\frac{\alpha m_{f_{1}}^{3}}{64 \pi^{4}}\left(\left|c_{L f_{1} f_{2} \gamma}\right|^{2}+\left|c_{R f_{1} f_{2} \gamma}\right|^{2}\right), \\
\Gamma\left(f_{1} \rightarrow f_{2} g\right) & =\frac{\alpha_{s} m_{f_{1}}^{3}}{48 \pi^{4}}\left(\left|c_{L f_{1} f_{2} g}\right|^{2}+\left|c_{R f_{1} f_{2} g}\right|^{2}\right) .
\end{aligned}
$$

We note in passing that similarly to the case of $\Delta F=2$ observables, in models where $S$ is a member of a larger electroweak multiplet additional contributions can arise from one-loop exchange of the associated charged scalars. However, contrary to the $\Delta F=2$ case, here such effects are not formally suppressed by either higher loop order or higher power in $1 / \Lambda$ expansion. In fact it is well known, that within THDMs charged Higgs contributions typically provide the largest effects on radiative decays (cf. [110]). In view of this, the bounds derived from dipole transitions can be viewed in such context as conservative estimates and stronger constraints are generically expected.

Imposing (2.1) together with stringent bounds on radiative LFV decays of heavy leptons allows to derive strong constraints on all $Y_{\ell_{i}, \ell_{j}}$. On the other hand, due to existing $\Delta F=2$ bounds on $Y_{q_{i} q_{j}}$, radiative decays of hadrons do not generally impose additional constraints. An exception are the $b \rightarrow s \gamma$ and $b \rightarrow s g$ transitions in the $B$ sector, as well as $s \rightarrow d g$ transitions in the kaon sector, which we thus examine in detail.

The $b \rightarrow s \gamma$ and $b \rightarrow s g$ transitions are severily constrained by the measurements of the inclusive $B \rightarrow X_{s} \gamma$ branching fraction and more generally, a global analysis of the inclusive and exclusive $b \rightarrow s \gamma$ and $b \rightarrow s \ell^{+} \ell^{-}$FCNC mediated decays. For the case of $B \rightarrow X_{s} \gamma$ one can write [111]

$$
\mathcal{B}\left(B \rightarrow X_{s} \gamma\right) \times 10^{4}=(3.36 \pm 0.23)-8.22 \operatorname{Re}\left(\Delta C_{7}\right)-1.99 \operatorname{Re}\left(\Delta C_{8}\right),
$$

where the terms quadratic in NP contributions and tiny effects from their imaginary parts have been neglected. $\Delta C_{7,8}$ are in turn defined as [110]

$$
\Delta C_{7}=\frac{c_{R b s \gamma}}{\sqrt{2} m_{b} G_{F} V_{t b}^{*} V_{t s}}, \quad \Delta C_{8}=\frac{c_{R b s g}}{\sqrt{2} m_{b} G_{F} V_{t b}^{*} V_{t s}} .
$$

Equation (4.44) holds at the renormalization scale $\mu_{0}=160 \mathrm{GeV}$. In our analysis we neglect small QCD running effects from our effective field theory (EFT) cut-off scale $\Lambda_{i}=1 \mathrm{TeV}$. The teoretical prediction in (4.44) has to be compared to the current experimental world 


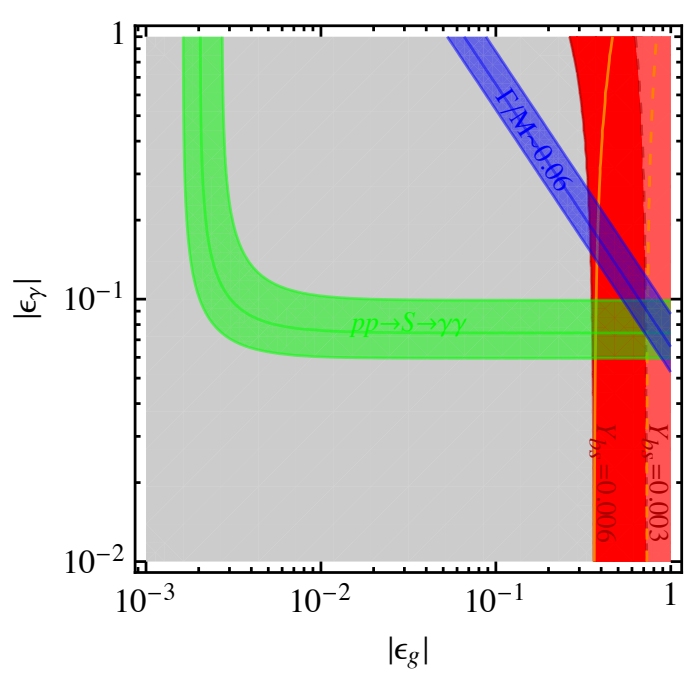

Figure 3. Combination of bounds on the $S$ couplings to photons and gluons coming from: the observation of $S \rightarrow \gamma$ events at the LHC (green band assuming gluon fusion dominated $S$ production), the ATLAS inferred $S$ resonance width (in blue band assuming a possible third decay channel saturating the width) and $b \rightarrow s \gamma$ constraints (in red bands) for various values of $S$ couplings to $b \bar{s}\left(Y_{b s}\right)$ allowed by $B_{s}$ oscillation measurements. The dark red (orange) contours refer to positive (negative) relative sign between $\epsilon_{\gamma}$ and $\epsilon_{g}$. See text for details.

average of $\mathcal{B}\left(B \rightarrow X_{s} \gamma\right)_{\exp }=(3.43 \pm 0.21 \pm 0.07) \times 10^{-4}$ [112]. Combined with the bounds from $B_{s}$ oscillations and (2.1), this leads to the $2 \sigma$ constraints shown in figure 3 . We observe that if $Y_{b s}$ is close to the bound set by $B_{s}$ oscillation measurements, a large $S$ width in (2.2) cannot be reproduced with only the $S \rightarrow g g$ mode.

On the other hand $c_{L b s \gamma}$ and $c_{L b s g}$ are most stringently constrained by a global fit to $b \rightarrow s \gamma$ and $b \rightarrow s \ell^{+} \ell^{-}$related measurements (cf. [113]). In particular one obtains

$$
C_{7}^{\prime}=\frac{c_{L b s \gamma}}{\sqrt{2} m_{b} G_{F} V_{t b}^{*} V_{t s}}, \quad C_{8}^{\prime}=\frac{c_{L b s g}}{\sqrt{2} m_{b} G_{F} V_{t b}^{*} V_{t s}},
$$

where a recent analysis [114] assuming real $C_{7,8}^{\prime}$ yields a constraint on the combination

$$
-0.1<\eta_{s b} \operatorname{Re}\left(C_{7}^{\prime}+0.24 C_{8}^{\prime}\right)<0.12 \text { at } 95 \% \text { C.L. },
$$

where $\eta_{s b} \simeq 0.58$ accounts for the QCD running between $\mu_{0}$ and the $b$-quark mass scale [113] . In this case the constraints are milder and any value of $Y_{s b}$ below the bound set by $B_{s}$ oscillation measurements does not significantly constrain the phenomenology of $S$.

In the kaon sector, dipole operators mediating $s \rightarrow d g$ transitions can be probed via the measurements of direct CP violation in $K^{0} \rightarrow 2 \pi$ decays, in particular $\epsilon^{\prime} / \epsilon$. Interestingly, the most recent SM prediction for this quantity [115]

$$
\left(\epsilon^{\prime} / \epsilon\right)_{\mathrm{SM}}=(1.9 \pm 4.5) \times 10^{-4},
$$

exhibits $\mathrm{a} \sim 2 \sigma$ tension with the corresponding experimental measurements [116-118] yielding

$$
\left(\epsilon^{\prime} / \epsilon\right)_{\exp }=(16.6 \pm 2.3) \times 10^{-4} .
$$


In turn, loop induced $S$ contributions to $c_{L s d g}, c_{L d s g}$ could play a role in bridging the gap between experiment and the SM expectations. The relevant effect in $\epsilon^{\prime} / \epsilon$ can be written as (cf. [115])

$$
\left|\epsilon^{\prime} / \epsilon\right|_{c_{L s d g}}=\frac{w}{\sqrt{2}\left|\epsilon_{K}\right| \operatorname{Re} A_{0}}\left|\operatorname{Im} A_{0}\right|_{c_{L s d g}}, \quad\left|\operatorname{Im} A_{0}\right|_{c_{L s d g}}=\left|\eta_{s d} \operatorname{Im}\left(c_{L s d g}\right)\left\langle(2 \pi)_{I=0}\left|Q_{L s d g}\right| K^{0}\right\rangle\right|,
$$

and analogously for $c_{L d s g}$. In our numerical evaluation we employ $1 / w=22.46,\left|\epsilon_{K}\right|=$ $1.596 \times 10^{-3}, \operatorname{Re} A_{0}=33.22 \times 10^{-8} \mathrm{GeV}$, while $\eta_{s d}=0.88$ accounts for the QCD running of $c_{L s d g}$ from the matching scale to $m_{c}$ [119]. The value of relevant matrix element is still fairly uncertain. A recent estimate [115] using a chiral quark model gives

$$
\left\langle(2 \pi)_{I=0}\left|Q_{L s d g}\right| K^{0}\right\rangle \simeq[2-8] \times 10^{-2} \mathrm{GeV}^{2} .
$$

Conservatively using the lower boundary of this interval and combining the remaining theoretical and experimental uncertainties in quadrature, we obtain a $2 \sigma$ preferred range of $\epsilon_{g} \operatorname{Im} Y_{s d}$ of

$$
1.4 \times 10^{-6}<\left|\epsilon_{g} \operatorname{Im} Y_{s d}\right|<7.5 \times 10^{-6} .
$$

Notice that such values can be consistent with constraints coming from neutral kaon oscillations provided $\left|\epsilon_{g}\right| \gtrsim 0.01$. Analogous conclusions hold for the product $\tilde{\epsilon}_{g} \operatorname{Re} Y_{s d}$ as well as $\tilde{\epsilon}_{g} \operatorname{Re} Y_{d s}$ or $\epsilon_{g} \operatorname{Im} Y_{d s}$.

Finally, we note that all couplings of $S$ to the top quark are only very weakly constrained. In particular radiative $t \rightarrow q \gamma$ and $t \rightarrow q g$ decays do not impose any relevant constraints on $Y_{t q}, Y_{q t}$. More information can in fact be extracted from existing LHC flavor tagged analyses at high $p_{T}$. While there are not current searches for singly produced $t j$ resonances, we can recast the recent ATLAS searches $[120,121]$ for $t \bar{b}$ resonances taking into account the poorer efficiency for $b$-tagging charmed jets ( 0.2 versus 0.7 for $b$-jets) but neglecting possible differences in acceptance and efficiency due to different production $(g g$ versus $\bar{q} q^{\prime}$ ). In the narrow width approximation, which applies for $\Gamma / M \ll 0.1$ the bound is

$$
\frac{\Gamma(S \rightarrow t \bar{c})}{\Gamma(S \rightarrow \gamma \gamma)} \lesssim 1500 \times\left(\frac{6 \mathrm{fb}}{\sigma(p p \rightarrow S)_{13 \mathrm{TeV} \mathcal{B}(S \rightarrow \gamma \gamma)}}\right)\left(\frac{R_{13 \mathrm{TeV} / 8 \mathrm{TeV}}}{5}\right)
$$

where $R_{13 \mathrm{TeV} / 8 \mathrm{TeV}} \equiv \sigma(p p \rightarrow S)_{13 \mathrm{TeV}} / \sigma(p p \rightarrow S)_{8 \mathrm{TeV}}$. On the other hand currently no relevant experimental bound on $\Gamma(S \rightarrow t \bar{u})$ can be extracted in this way .

\subsection{Implications of $S \rightarrow \gamma \gamma$ for $R_{K}$ puzzle}

Recently, the $\mathrm{LHCb}$ collaboration has reported discrepancies in angular observables in the rare $b \rightarrow s \mu^{+} \mu^{-}$FCNC mediated decay $B \rightarrow K^{*} \mu^{+} \mu^{-}[122,123]$ as well as in the ratio of decay rates of $B^{+} \rightarrow K^{+} \mu^{+} \mu^{-}$versus $B^{+} \rightarrow K^{+} e^{+} e^{-}$[124]. Interestingly, both anomalies can be successfully accommodated by supplementing the SM contributions with

$$
\mathcal{H}_{\mathrm{eff}}=\left[\bar{s} \gamma^{\mu}\left(c P_{L}+c^{\prime} P_{R}\right) b\right]\left[\bar{\mu} \gamma_{\mu}\left(v+a \gamma_{5}\right) \mu\right],
$$

where a good fit to all relevant $b \rightarrow s \mu^{+} \mu^{-}$data can be obtained for several choices of non-vanishing parameters $[114,125]$

$$
\left[c v \simeq(34 \mathrm{TeV})^{-2}\right] \quad \text { or } \quad\left[c v=-c a \simeq(48 \mathrm{TeV})^{-2}\right] \quad \text { or } \quad\left[c^{\prime} v=-c v \simeq(35 \mathrm{TeV})^{-2}\right] .
$$


Such contributions cannot be generated via tree-level exchange of a neutral scalar. Instead, perhaps the simplest explanation is due the exchange of a neutral massive vector $\mathrm{U}(1)^{\prime}$ gauge boson $\left(Z^{\prime}\right)[126,127]$. Interestingly such models, if weakly coupled, require the introduction of scalars neutral under SM gauge group as well as heavy vector-like quark and lepton partners. The scalar condensate controls both the spontaneous breaking of the $\mathrm{U}(1)^{\prime}$ as well as the mixing of SM fermions with their vector-like counterparts, which endows them with couplings to the $Z^{\prime}$.

To examine the possibility that $S$ represents the physical excitation of the $\mathrm{U}(1)^{\prime}$ breaking condensate we consider the following simple model (see $[127,128]$ for related previous work). We introduce two pairs of vector-like fermions $\mathcal{Q}$ and $\Psi$ with $\mathrm{SM}$ and $\mathrm{U}(1)^{\prime}$ gauge quantum numbers $\left(3,2,1 / 6, Q^{\prime}\right)$ and $\left(1,2,-1, Q^{\prime}\right)$ as well as $\mathcal{X}=(w+S) / \sqrt{2}$ carrying $\left(1,1,0, Q^{\prime}\right)$ and with $w \equiv \sqrt{2}\langle\mathcal{X}\rangle$. All SM fields are assumed to be neutral under $\mathrm{U}(1)^{\prime}$. For any nonvanishing $\mathrm{U}(1)^{\prime}$ charge $Q^{\prime}$, the only terms coupling SM fermions to the new sector are the $\mathcal{X}$ Yukawa couplings between $\mathcal{Q}, \Psi$ and the corresponding SM fermion doublets $q$ and $\ell$

$$
\mathcal{L}_{\text {mix }}=-\tilde{Y}_{q} \overline{\mathcal{Q}} \mathcal{X} q-\tilde{Y}_{\ell} \bar{\Psi} \mathcal{X} \ell .
$$

If they mix with a single SM generation the weak and mass eigenstates will be related via $[129,130]$

$$
\left(\begin{array}{l}
q \\
\mathcal{Q}
\end{array}\right)=\left(\begin{array}{cc}
\cos \theta & \sin \theta_{q} \\
-\sin \theta_{q} & \cos \theta_{q}
\end{array}\right)\left(\begin{array}{c}
q^{\prime} \\
\mathcal{Q}^{\prime}
\end{array}\right)
$$

where

$$
\tan 2 \theta_{q}=\frac{\sqrt{2} M_{\mathcal{Q}} w \tilde{Y}_{q}}{M_{\mathcal{Q}}^{2}-\left(w \tilde{Y}_{q}\right)^{2} / 2-\left(v y_{q}\right)^{2} / 2},
$$

where $M_{\mathcal{Q}}$ is the $\mathcal{Q}$ Dirac mass, $y_{q}$ is the SM fermion Yukawa coupling to the Higgs and $v=246 \mathrm{GeV}$. The mass eigenstates are then determined via

$$
m_{\mathcal{Q}} m_{q}=M_{\mathcal{Q}} y_{q} \frac{v}{\sqrt{2}}, \quad m_{q}^{2}+m_{\mathcal{Q}}^{2}=M_{\mathcal{Q}}^{2}+\frac{w^{2}}{2} \tilde{Y}_{q}^{2}+\frac{v^{2}}{2} y_{q}^{2} .
$$

The analogous expressions for leptons can be obtained via the replacement $q \rightarrow \ell$. Note that due to the different up- and down-type quark masses within the SM weak doublet, $\theta_{q}$ and $m_{\mathcal{Q}}$ can differ between the up and down sectors. In practice however the differences are negligible for $M_{\mathcal{Q}} \gtrsim 1 \mathrm{TeV}$. Secondly, since the SM fermion mixes with a state with exactly the same SM quantum numbers, modifications of $W$ and $Z$ couplings to the physical state $q$ are protected by both EW and $\mathrm{U}(1)^{\prime}$ breaking and thus safely small. Together, both effects make this model largely transparent to EW precision tests. Consequently even maximal mixing is perfectly allowed. It does however significantly affect $\mathrm{U}(1)^{\prime}$ and $S$ interactions. In particular, mixing induces perturbative $(\mathcal{Q}, q)$ loop contributions to radiative $S$ decays. Secondly, it provides new decay channels for $S$ : for example if $\Psi$ is lighter than $S$, the later can decay to a $\Psi \ell$ pair. Such decay rates are proportional to $\left(\tilde{Y}_{\ell} \cos \theta_{\ell}\right)^{2}$.

Finally, the induced $\mathrm{U}(1)^{\prime}$ charges of $q$ and $\ell$ can have effects in low energy processes of SM fermions. In particular, if we furthermore assume, that $\mathcal{Q}$ only mixes with the third generation SM doublet in the up-quark mass basis, where $q=\left(t_{L}, \sum_{i} V_{t i} d_{L}^{i}\right)^{T}$, while $\Psi$ 
only mixes with the second generation SM doublet in the charged lepton mass basis, where $\ell=\left(\sum_{i} U_{i \mu} \nu_{i}, \mu_{L}\right)^{T}$ we obtain

$$
c v=-c a=\frac{\sin ^{2} \theta_{q} \sin ^{2} \theta_{\ell}\left|V_{t b}^{*} V_{t s}\right|}{w^{2}} .
$$

We see that for $w \lesssim 1 \mathrm{TeV}$ a preferred set of values for $c v, c a$ can in principle be reproduced. However, the same interactions also generate contributions to $B_{s}$ mixing. In particular [127]

$$
\frac{M_{12}}{M_{12}^{\mathrm{SM}}}=1+\Lambda_{\mathrm{SM}}^{2} \sin ^{4} \theta_{q}\left(V_{t b}^{*} V_{t s}\right)^{2}\left(\frac{1}{w^{2}}+\frac{1}{16 \pi^{2}} \frac{1}{M_{\mathcal{Q}}^{2}}\right)
$$

where $\Lambda_{\mathrm{SM}}=4 \pi m_{W} / g_{W} V_{t b}^{*} V_{t s} \sqrt{S_{0}} \simeq 40 \mathrm{TeV}$. For $M_{\mathcal{Q}} \gtrsim 1 \mathrm{TeV}$, the second term in the brackets is negligible. The same effect is present in $B_{d}$ mixing, by replacing $V_{t s} \rightarrow V_{t d}$. Requiring $\left|M_{12} / M_{12}^{\mathrm{SM}}-1\right|<0.15$ thus constrains $w / \sin ^{2} \theta_{q} \gtrsim 4 \mathrm{TeV}$. This condition can be easily satisfied for even for $\tilde{Y}_{q} \sim 1$ at $M_{\mathcal{Q}} \sim 1 \mathrm{TeV}$ provided a low enough $\mathrm{U}(1)^{\prime}$ breaking $w \lesssim 0.5 \mathrm{TeV}$. Note that for $M=750 \mathrm{GeV}$, this corresponds to a $\mathcal{X}$ with a quartic value of $\sim 1$.

On the other hand neutrino trident production constrains the $Z^{\prime}$ couplings to muons. In our case, the relevant cross-section, normalized to the SM value is given by [131]

$$
\frac{\sigma}{\sigma_{\mathrm{SM}}} \simeq \frac{\left(1+2 v^{2} \sin ^{2} \theta_{\ell} / w^{2}\right)^{2}+\left(1+4 s_{W}^{2}+2 v^{2} \sin ^{2} \theta_{\ell} / w^{2}\right)}{1+\left(1+4 s_{W}^{2}\right)^{2}}
$$

where $s_{W} \simeq \sqrt{0.23}$ is the sine of the weak mixing angle. Comparing this with a recently compiled average of measurements $\sigma_{\exp } / \sigma_{\mathrm{SM}}=0.83 \pm 0.18$ [127] we obtain a $2 \sigma$ bound on $w / \sin \theta_{\ell} \gtrsim 0.91 \mathrm{TeV} .^{8}$

The results of a combined fit to $S$ signal strength and flavor data is shown in figure 4, where we also fix $\epsilon_{\gamma}=\epsilon_{g}=0.1$ to reproduce the required radiative $S$ widths to within $50 \%$ (shown in blue shading). In addition requiring to reproduce $b \rightarrow s \mu^{+} \mu^{-}$data, the constraint coming from $B_{s}$ oscillation measurements and neutrino trident production exclude the red and orange shaded regions at small and large $\theta_{\ell}$, respectively. The $S$ width is dominated by the decays $S \rightarrow g g$, however at small $M_{\Psi}$ also $S \rightarrow \mu^{ \pm} \Psi^{\mp}$ and $S \rightarrow \nu \Psi^{0}$ can become important. The invisible decay mode provides a constraint on this setup [77] (shown in brown shading in the figure). Consequently the ATLAS indicated large $S$ width cannot be obtained with typical allowed widths of the order of $\Gamma / M \lesssim 0.002$. The charged $\Psi$ state is radiatively split in mass from the neutral component [132-134] and will decay promptly as $\Psi^{ \pm} \rightarrow \pi^{ \pm} \Psi^{0}$. Since the pion in the decay is very soft and can be missed, the resulting signature for $S \rightarrow \mu \Psi$ resembles a monolepton, but with an additional soft pion.

Since low energy flavor observables do not directly depend on the $\mathrm{U}(1)^{\prime}$ gauge coupling, the mass of the corresponding gauge boson is undetermined. If it is light enough $S$ can decay to it, providing additional interesting decay modes $S \rightarrow 2 A^{\prime} \rightarrow 4 j_{b}$ and $S \rightarrow 2 A^{\prime} \rightarrow 4 \mu$. Finally, in the narrow window where $M>2 m_{A^{\prime}}>2 M_{\Psi}$, also $S \rightarrow 2 A^{\prime} \rightarrow 2 \Psi^{ \pm} 2 \mu$ decay mode is possible, while $S \rightarrow 2 A^{\prime} \rightarrow 2 \Psi^{0} 2 \nu$ just contributes to the invisible $S$ width.

\footnotetext{
${ }^{8}$ This bound makes the $Z^{\prime}$ contribution to $a_{\mu}$ negligible in this model.
} 


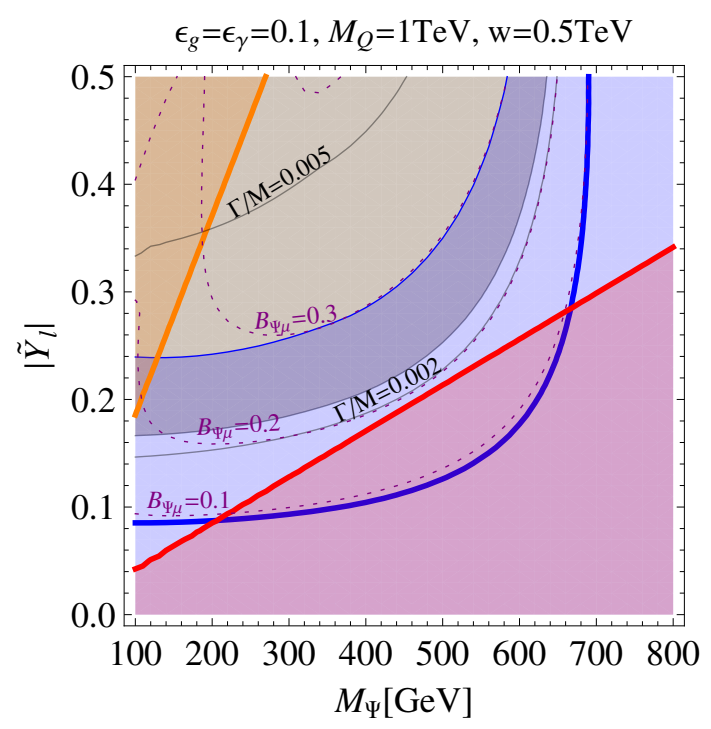

Figure 4. Constraints on the parameter space of the gauged $\mathrm{U}(1)^{\prime}$ model addressing the observed LHCb anomalies in $B \rightarrow K^{(*)} \mu^{+} \mu^{-}$decays: hints of $S$ production and decay to two photons at $13 \mathrm{TeV}$ LHC (central value in thick blue line, blue band allows for $50 \%$ variation in signal strength), monojet bounds at $8 \mathrm{TeV}$ LHC (excluded region in brown), $B_{s}-\bar{B}_{s}$ oscillation measurements (excluded region in red), neutrino trident production (excluded region in orange). Overlaid are contours of constant $\Gamma / M$ (in full black lines) and $\mathcal{B}_{\Psi \mu} \equiv \mathcal{B}\left(S \rightarrow \Psi^{ \pm} \mu^{\mp}\right)$ (in purple dotted lines). See text for details.

On the other hand, the new vector-like fermions in the model can be searched for directly at colliders, such as the LHC. Limits on corresponding vector-like quarks are currently at $\mathcal{O}(800-900) \mathrm{GeV}$ [135-141], while the prospects for the high-luminosity LHC (HL-LHC) at $14 \mathrm{TeV}$ are around $1500 \mathrm{GeV}[142,143]$. Turning to the lepton sector, constraints reported in the PDG stem so far from LEP, and are at $m_{L} \gtrsim 100 \mathrm{GeV}$ [85]. Regarding the LHC, existing multi-lepton searches using the full $\sqrt{s}=8 \mathrm{TeV}$ data set can be used to place limits on masses of vector-like leptons. These are rather model dependent and currently reside at around $\mathcal{O}(200-400) \mathrm{GeV}$ [144]. In turn, the some of the interesting parameter space of the model should be accessible with the next LHC run and finally at the HL-LHC.

\section{Conclusions}

We have explored the potential physics underlying the excess in searches for di-photon resonances at ATLAS and CMS, parameterizing it in terms of higher dimensional operators including the SM fields and a new (pseudo-)scalar particle $S .{ }^{9}$ Beyond that, we considered renormalizable interactions of the latter with new vector-like fermions. In particular, we have addressed the question of calculability in scenarios where the tentative signal is mediated by loops of such new fermions, indicating that existing (apparent) explanations featuring sizable $S$ Yukawa couplings and a bunch of new fermions are in fact not calculable in perturbation theory. In particular, we have proposed a simple strategy

\footnotetext{
${ }^{9}$ While we focused on the case of $S$ being an SM singlet, we also commented on the electroweak-doublet hypothesis.
} 
to test if in a given model perturbation theory can be trusted. We applied this to a toy model of new vector-like fermions and derived concrete bounds on their number and their Yukawa couplings, confronting them with the parameter space of possible explanations of the anomaly.

Moreover, we have studied correlations between the di-photon excess and low energy observables. A first focus was on dipole moments of SM fermions coupled to $S$, which are generated due to the (sizable) coupling of $S$ to photons (and gluons). We showed that the constraints in this sector are able to shed light on the interactions of $S$. In particular, we demonstrated that an explanation of the muon MDM anomaly requires a $S \mu^{+} \mu^{-}$coupling of $\mathcal{O}(0.1)$ and can be tested by limits on the decay $S \rightarrow \mu^{+} \mu^{-}$. The latter are already cutting significantly into the parameter space and will explore a large portion of it with the next LHC run. On the other hand, upper bounds on $a_{e}$ as well as on the corresponding electric dipole moment can significantly constrain the couplings of $S$ to electrons.

After that, we turned to correlations between the di-photon excess and flavor physics, considering four-fermion operators generated after integrating out $S$ at the tree level as well as radiatively generated dipole operators due to the $S \gamma \gamma$ and $S g g$ interactions. We consequently presented limits on the $S$ Yukawa couplings from measurements in the flavor sector, such as meson oscillations as well as FCNC decays of hadrons and leptons. While in the quark case, usually limits from meson mixing provide the most stringent constraints, we showed in particular that in the presence of new right-handed contributions to dipole operators, bounds on $b \rightarrow s \gamma$ transitions, together with the requirement to reproduce the correct $S$ width, can add non-trivial information on the Yukawa couplings. We explored these constraints, together with those from the cross section of the $S \rightarrow \gamma \gamma$ signal and from $B_{s}$ oscillations in the $\epsilon_{\gamma}, \epsilon_{g}, Y_{s b} / Y_{b s}$ parameter space. Similarly interesting interplay occurs in the kaon sector, where we have shown that $\epsilon_{g}, Y_{s d} / Y_{d s}$ effects consistent with bounds from neutral kaon oscillation measurements could ameliorate the tension between the recent SM predictions and experimental measurements of the direct $\mathrm{CP}$ violation in $K^{0} \rightarrow 2 \pi$ decays $\left(\epsilon^{\prime} / \epsilon\right)$.

Finally, we also considered the flavor anomalies recent found in the angular analysis of $B \rightarrow K^{*} \mu^{+} \mu^{-}$and in $R_{K}$. We showed that the scalar $S$ could be linked to the breaking of a gauged $\mathrm{U}(1)^{\prime}$ symmetry, which in turn could address these anomalies via $Z^{\prime}$ exchange. We assumed the latter being coupled to new vector-like fermions, charged under the $\mathrm{U}(1)^{\prime}$, that mix with the $\left(\mathrm{U}(1)^{\prime}\right.$ neutral) SM fermions only via the vacuum expectation value of a scalar that features $S$ as a physical excitation. We considered various constraints on this model, summarized in figure 4 .

\section{Acknowledgments}

We thank Luca Di Luzio for useful discussions. Special thanks goes also to Ulrich Haisch for his several enlightening comments on the first version of the manuscript. JFK acknowledges the financial support from the Slovenian Research Agency (research core funding No. P10035). FG acknowledges support by a Marie Curie Intra European Fellowship within the EU FP7 (grant No. PIEF-GA-2013-628224). MN acknowledges support of the STFC grant ST/L000385/1, and King's College, Cambridge. 
Note added. Our paper appeared on arXiv on the same day as [145]. This reference also analyzes calculability and perturbativity constraints on possible explanations of the $750 \mathrm{GeV}$ diphoton excess. Note, however, that our paper uses a different criterion for calculability.

Open Access. This article is distributed under the terms of the Creative Commons Attribution License (CC-BY 4.0), which permits any use, distribution and reproduction in any medium, provided the original author(s) and source are credited.

\section{References}

[1] ATLAS collaboration, Search for resonances decaying to photon pairs in $3.2 \mathrm{fb}^{-1}$ of $p p$ collisions at $\sqrt{s}=13 \mathrm{TeV}$ with the ATLAS detector, ATLAS-CONF-2015-081.

[2] CMS collaboration, Search for new physics in high mass diphoton events in proton-proton collisions at $13 \mathrm{TeV}$, CMS-PAS-EXO-15-004.

[3] ATLAS collaboration, Search for high-mass diphoton resonances in pp collisions at $\sqrt{s}=8 \mathrm{TeV}$ with the ATLAS detector, Phys. Rev. D 92 (2015) 032004 [arXiv:1504.05511] [INSPIRE].

[4] CMS collaboration, Search for High-Mass Diphoton Resonances in pp Collisions at $\sqrt{s}=8 \mathrm{TeV}$ with the CMS Detector, CMS-PAS-EXO-12-045.

[5] A. Pilaftsis, Diphoton Signatures from Heavy Axion Decays at the CERN Large Hadron Collider, Phys. Rev. D 93 (2016) 015017 [arXiv: 1512.04931] [InSPIRE].

[6] S. Knapen, T. Melia, M. Papucci and K. Zurek, Rays of light from the LHC, Phys. Rev. D 93 (2016) 075020 [arXiv: 1512.04928] [InSPIRE].

[7] A. Angelescu, A. Djouadi and G. Moreau, Scenarii for interpretations of the LHC diphoton excess: two Higgs doublets and vector-like quarks and leptons, Phys. Lett. B 756 (2016) 126 [arXiv: 1512.04921] [INSPIRE].

[8] S. Di Chiara, L. Marzola and M. Raidal, First interpretation of the $750 \mathrm{GeV}$ diphoton resonance at the LHC, Phys. Rev. D 93 (2016) 095018 [arXiv:1512.04939] [InSPIRE].

[9] D. Buttazzo, A. Greljo and D. Marzocca, Knocking on new physics' door with a scalar resonance, Eur. Phys. J. C 76 (2016) 116 [arXiv:1512.04929] [INSPIRE].

[10] M. Backovic, A. Mariotti and D. Redigolo, Di-photon excess illuminates Dark Matter, JHEP 03 (2016) 157 [arXiv: 1512.04917] [INSPIRE].

[11] K. Harigaya and Y. Nomura, Composite Models for the 750 GeV Diphoton Excess, Phys. Lett. B 754 (2016) 151 [arXiv: 1512.04850] [INSPIRE].

[12] Y. Mambrini, G. Arcadi and A. Djouadi, The LHC diphoton resonance and dark matter, Phys. Lett. B 755 (2016) 426 [arXiv:1512.04913] [INSPIRE].

[13] A. Belyaev, G. Cacciapaglia, H. Cai, T. Flacke, A. Parolini and H. Serôdio, Singlets in Composite Higgs Models in light of the LHC di-photon searches, arXiv:1512.07242 [INSPIRE].

[14] G.M. Pelaggi, A. Strumia and E. Vigiani, Trinification can explain the di-photon and di-boson LHC anomalies, JHEP 03 (2016) 025 [arXiv: 1512.07225] [INSPIRE]. 
[15] U.K. Dey, S. Mohanty and G. Tomar, $750 \mathrm{GeV}$ resonance in the dark left-right model, Phys. Lett. B 756 (2016) 384 [arXiv: 1512.07212] [INSPIRE].

[16] A.E.C. Hernández and I. Nisandzic, LHC diphoton $750 \mathrm{GeV}$ resonance as an indication of $\mathrm{SU}(3)_{c} \times \mathrm{SU}(3)_{L} \times \mathrm{U}(1)_{X}$ gauge symmetry, arXiv:1512.07165 [INSPIRE].

[17] C.W. Murphy, Vector Leptoquarks and the $750 \mathrm{GeV}$ Diphoton Resonance at the LHC, Phys. Lett. B 757 (2016) 192 [arXiv:1512.06976] [INSPIRE].

[18] S.M. Boucenna, S. Morisi and A. Vicente, The LHC diphoton resonance from gauge symmetry, arXiv:1512.06878 [INSPIRE].

[19] J. de Blas, J. Santiago and R. Vega-Morales, New vector bosons and the diphoton excess, arXiv: 1512.07229 [INSPIRE].

[20] P.S.B. Dev and D. Teresi, Asymmetric Dark Matter in the Sun and the Diphoton Excess at the LHC, arXiv: 1512.07243 [INSPIRE].

[21] M. Chala, M. Duerr, F. Kahlhoefer and K. Schmidt-Hoberg, Tricking Landau-Yang: How to obtain the diphoton excess from a vector resonance, Phys. Lett. B 755 (2016) 145 [arXiv:1512.06833] [INSPIRE].

[22] M. Bauer and M. Neubert, Flavor Anomalies, the Diphoton Excess and a Dark Matter Candidate, arXiv:1512.06828 [INSPIRE].

[23] J.M. Cline and Z. Liu, LHC diphotons from electroweakly pair-produced composite pseudoscalars, arXiv:1512.06827 [INSPIRE].

[24] L. Berthier, J.M. Cline, W. Shepherd and M. Trott, Effective interpretations of a diphoton excess, JHEP 04 (2016) 084 [arXiv:1512.06799] [INSPIRE].

[25] J.S. Kim, K. Rolbiecki and R. Ruiz de Austri, Model-independent combination of diphoton constraints at $750 \mathrm{GeV}$, Eur. Phys. J. C 76 (2016) 251 [arXiv:1512.06797] [InSPIRE].

[26] X.-J. Bi, Q.-F. Xiang, P.-F. Yin and Z.-H. Yu, The $750 \mathrm{GeV}$ diphoton excess at the LHC and dark matter constraints, Nucl. Phys. B 909 (2016) 43 [arXiv:1512.06787] [INSPIRE].

[27] J.J. Heckman, 750 GeV Diphotons from a D3-brane, Nucl. Phys. B 906 (2016) 231 [arXiv: 1512.06773] [INSPIRE].

[28] F.P. Huang, C.S. Li, Z.L. Liu and Y. Wang, $750 \mathrm{GeV}$ Diphoton Excess from Cascade Decay, arXiv:1512.06732 [INSPIRE].

[29] J. Cao, C. Han, L. Shang, W. Su, J.M. Yang and Y. Zhang, Interpreting the $750 \mathrm{GeV}$ diphoton excess by the singlet extension of the Manohar-Wise model, Phys. Lett. B 755 (2016) 456 [arXiv: 1512.06728] [INSPIRE].

[30] F. Wang, L. Wu, J.M. Yang and M. Zhang, $750 \mathrm{GeV}$ Diphoton Resonance, $125 \mathrm{GeV}$ Higgs and Muon g-2 Anomaly in Deflected Anomaly Mediation SUSY Breaking Scenario, arXiv: 1512.06715 [INSPIRE].

[31] O. Antipin, M. Mojaza and F. Sannino, A natural Coleman-Weinberg theory explains the diphoton excess, arXiv:1512.06708 [INSPIRE].

[32] X.-F. Han and L. Wang, Implication of the $750 \mathrm{GeV}$ diphoton resonance on two-Higgs-doublet model and its extensions with Higgs field, Phys. Rev. D 93 (2016) 055027 [arXiv: 1512.06587] [INSPIRE].

[33] R. Ding, L. Huang, T. Li and B. Zhu, Interpreting $750 \mathrm{GeV}$ Diphoton Excess with R-parity Violating Supersymmetry, arXiv:1512.06560 [INSPIRE]. 
[34] W. Chao, Symmetries Behind the 750 GeV Diphoton Excess, arXiv:1512.06297 [InSPIRE].

[35] D. Barducci, A. Goudelis, S. Kulkarni and D. Sengupta, One jet to rule them all: monojet constraints and invisible decays of a $750 \mathrm{GeV}$ diphoton resonance, arXiv:1512.06842 [INSPIRE].

[36] W.S. Cho, D. Kim, K. Kong, S.H. Lim, K.T. Matchev, J.-C. Park et al., 750 GeV Diphoton Excess May Not Imply a 750 GeV Resonance, Phys. Rev. Lett. 116 (2016) 151805 [arXiv: 1512.06824] [INSPIRE].

[37] W. Liao and H.-q. Zheng, Scalar resonance at $750 \mathrm{GeV}$ as composite of heavy vector-like fermions, arXiv:1512.06741 [INSPIRE].

[38] T.-F. Feng, X.-Q. Li, H.-B. Zhang and S.-M. Zhao, The LHC $750 \mathrm{GeV}$ diphoton excess in supersymmetry with gauged baryon and lepton numbers, arXiv:1512.06696 [INSPIRE].

[39] D. Bardhan, D. Bhatia, A. Chakraborty, U. Maitra, S. Raychaudhuri and T. Samui, Radion Candidate for the LHC Diphoton Resonance, arXiv:1512.06674 [INSPIRE].

[40] J. Chang, K. Cheung and C.-T. Lu, Interpreting the $750 \mathrm{GeV}$ diphoton resonance using photon jets in hidden-valley-like models, Phys. Rev. D 93 (2016) 075013 [arXiv: 1512.06671] [INSPIRE].

[41] M.-x. Luo, K. Wang, T. Xu, L. Zhang and G. Zhu, Squarkonium, diquarkonium and octetonium at the LHC and their diphoton decays, Phys. Rev. D 93 (2016) 055042 [arXiv: 1512.06670] [INSPIRE].

[42] H. Han, S. Wang and S. Zheng, Scalar Explanation of Diphoton Excess at LHC, Nucl. Phys. B 907 (2016) 180 [arXiv: 1512.06562] [INSPIRE].

[43] S. Chang, A Simple U(1) Gauge Theory Explanation of the Diphoton Excess, Phys. Rev. D 93 (2016) 055016 [arXiv: 1512.06426] [INSPIRE].

[44] C. Han, H.M. Lee, M. Park and V. Sanz, The diphoton resonance as a gravity mediator of dark matter, Phys. Lett. B 755 (2016) 371 [arXiv:1512.06376] [INSPIRE].

[45] J. Bernon and C. Smith, Could the width of the diphoton anomaly signal a three-body decay?, Phys. Lett. B 757 (2016) 148 [arXiv:1512.06113] [INSPIRE].

[46] L.M. Carpenter, R. Colburn and J. Goodman, Supersoft SUSY Models and the $750 \mathrm{GeV}$ Diphoton Excess, Beyond Effective Operators, arXiv:1512.06107 [INSPIRE].

[47] E. Megias, O. Pujolàs and M. Quirós, On dilatons and the LHC diphoton excess, arXiv: 1512.06106 [INSPIRE].

[48] A. Alves, A.G. Dias and K. Sinha, The $750 \mathrm{GeV}$ S-cion: Where else should we look for it?, Phys. Lett. B 757 (2016) 39 [arXiv:1512.06091] [INSPIRE].

[49] E. Gabrielli, K. Kannike, B. Mele, M. Raidal, C. Spethmann and H. Veermäe, A SUSY Inspired Simplified Model for the $750 \mathrm{GeV}$ Diphoton Excess, Phys. Lett. B 756 (2016) 36 [arXiv: 1512.05961] [INSPIRE].

[50] J.S. Kim, J. Reuter, K. Rolbiecki and R. Ruiz de Austri, A resonance without resonance: scrutinizing the diphoton excess at $750 \mathrm{GeV}$, Phys. Lett. B 755 (2016) 403 [arXiv: 1512.06083] [INSPIRE].

[51] R. Benbrik, C.-H. Chen and T. Nomura, Higgs singlet boson as a diphoton resonance in a vectorlike quark model, Phys. Rev. D 93 (2016) 055034 [arXiv:1512.06028] [INSPIRE]. 
[52] Y. Bai, J. Berger and R. Lu, 750 GeV dark pion: Cousin of a dark G-parity odd WIMP, Phys. Rev. D 93 (2016) 076009 [arXiv:1512.05779] [InSPIRE].

[53] A. Falkowski, O. Slone and T. Volansky, Phenomenology of a $750 \mathrm{GeV}$ Singlet, JHEP 02 (2016) 152 [arXiv: 1512.05777] [inSPIRE].

[54] C. Csáki, J. Hubisz and J. Terning, Minimal model of a diphoton resonance: Production without gluon couplings, Phys. Rev. D 93 (2016) 035002 [arXiv:1512.05776] [INSPIRE].

[55] J. Chakrabortty, A. Choudhury, P. Ghosh, S. Mondal and T. Srivastava, Di-photon resonance around $750 \mathrm{GeV}$ : shedding light on the theory underneath, arXiv:1512.05767 [INSPIRE].

[56] L. Bian, N. Chen, D. Liu and J. Shu, Hidden confining world on the $750 \mathrm{GeV}$ diphoton excess, Phys. Rev. D 93 (2016) 095011 [arXiv:1512.05759] [InSPIRE].

[57] D. Curtin and C.B. Verhaaren, Quirky Explanations for the Diphoton Excess, Phys. Rev. D 93 (2016) 055011 [arXiv: 1512.05753] [INSPIRE].

[58] S. Fichet, G. von Gersdorff and C. Royon, Scattering light by light at $750 \mathrm{GeV}$ at the LHC, Phys. Rev. D 93 (2016) 075031 [arXiv: 1512.05751] [INSPIRE].

[59] W. Chao, R. Huo and J.-H. Yu, The Minimal Scalar-Stealth Top Interpretation of the Diphoton Excess, arXiv:1512.05738 [INSPIRE].

[60] S.V. Demidov and D.S. Gorbunov, On the sgoldstino interpretation of the diphoton excess, JETP Lett. 103 (2016) 219 [arXiv:1512.05723] [INSPIRE].

[61] J.M. No, V. Sanz and J. Setford, See-saw composite Higgs model at the LHC: Linking naturalness to the $750 \mathrm{GeV}$ diphoton resonance, Phys. Rev. D 93 (2016) 095010 [arXiv: 1512.05700] [INSPIRE].

[62] D. Bečirević, E. Bertuzzo, O. Sumensari and R. Zukanovich Funchal, Can the new resonance at LHC be a CP-Odd Higgs boson?, Phys. Lett. B 757 (2016) 261 [arXiv: 1512.05623] [INSPIRE].

[63] A. Ahmed, B.M. Dillon, B. Grzadkowski, J.F. Gunion and Y. Jiang, Higgs-radion interpretation of $750 \mathrm{GeV}$ di-photon excess at the LHC, arXiv:1512.05771 [INSPIRE].

[64] P. Cox, A.D. Medina, T.S. Ray and A. Spray, Diphoton Excess at $750 \mathrm{GeV}$ from a Radion in the Bulk-Higgs Scenario, arXiv:1512.05618 [INSPIRE].

[65] A. Kobakhidze, F. Wang, L. Wu, J.M. Yang and M. Zhang, $750 \mathrm{GeV}$ diphoton resonance in a top and bottom seesaw model, Phys. Lett. B 757 (2016) 92 [arXiv:1512.05585] [INSPIRE].

[66] Q.-H. Cao, Y. Liu, K.-P. Xie, B. Yan and D.-M. Zhang, A Boost Test of Anomalous Diphoton Resonance at the $L H C$, arXiv:1512.05542 [INSPIRE].

[67] B. Dutta, Y. Gao, T. Ghosh, I. Gogoladze and T. Li, Interpretation of the diphoton excess at CMS and ATLAS, Phys. Rev. D 93 (2016) 055032 [arXiv:1512.05439] [INSPIRE].

[68] C. Petersson and R. Torre, 750 GeV Diphoton Excess from the Goldstino Superpartner, Phys. Rev. Lett. 116 (2016) 151804 [arXiv:1512.05333] [INSPIRE].

[69] M. Low, A. Tesi and L.-T. Wang, A pseudoscalar decaying to photon pairs in the early LHC Run 2 data, JHEP 03 (2016) 108 [arXiv:1512.05328] [INSPIRE].

[70] S.D. McDermott, P. Meade and H. Ramani, Singlet Scalar Resonances and the Diphoton Excess, Phys. Lett. B 755 (2016) 353 [arXiv:1512.05326] [INSPIRE]. 
[71] T. Higaki, K.S. Jeong, N. Kitajima and F. Takahashi, The QCD Axion from Aligned Axions and Diphoton Excess, Phys. Lett. B 755 (2016) 13 [arXiv:1512.05295] [INSPIRE].

[72] B. Bellazzini, R. Franceschini, F. Sala and J. Serra, Goldstones in Diphotons, JHEP 04 (2016) 072 [arXiv: 1512.05330] [INSPIRE].

[73] E. Molinaro, F. Sannino and N. Vignaroli, Minimal Composite Dynamics versus Axion Origin of the Diphoton excess, arXiv:1512.05334 [INSPIRE].

[74] K.M. Patel and P. Sharma, Interpreting $750 \mathrm{GeV}$ diphoton excess in $\mathrm{SU}(5)$ grand unified theory, Phys. Lett. B 757 (2016) 282 [arXiv:1512.07468] [INSPIRE].

[75] Y. Nakai, R. Sato and K. Tobioka, Footprints of New Strong Dynamics via Anomaly and the $750 \mathrm{GeV}$ Diphoton, Phys. Rev. Lett. 116 (2016) 151802 [arXiv:1512.04924] [INSPIRE].

[76] K. Das and S.K. Rai, $750 \mathrm{GeV}$ diphoton excess in a U(1) hidden symmetry model, Phys. Rev. D 93 (2016) 095007 [arXiv: 1512.07789] [INSPIRE].

[77] R. Franceschini et al., What is the $\gamma \gamma$ resonance at $750 \mathrm{GeV}$ ?, JHEP 03 (2016) 144 [arXiv:1512.04933] [INSPIRE].

[78] M.E. Machacek and M.T. Vaughn, Two Loop Renormalization Group Equations in a General Quantum Field Theory. 1. Wave Function Renormalization, Nucl. Phys. B 222 (1983) 83 [INSPIRE].

[79] M.E. Machacek and M.T. Vaughn, Two Loop Renormalization Group Equations in a General Quantum Field Theory. 2. Yukawa Couplings, Nucl. Phys. B 236 (1984) 221 [INSPIRE].

[80] M.E. Machacek and M.T. Vaughn, Two Loop Renormalization Group Equations in a General Quantum Field Theory. 3. Scalar Quartic Couplings, Nucl. Phys. B 249 (1985) 70 [INSPIRE].

[81] M.-x. Luo, H.-w. Wang and Y. Xiao, Two loop renormalization group equations in general gauge field theories, Phys. Rev. D 67 (2003) 065019 [hep-ph/0211440] [InSPIRE].

[82] ATLAS collaboration, Search for new phenomena in the dijet mass distribution using pp collision data at $\sqrt{s}=8 \mathrm{TeV}$ with the ATLAS detector, Phys. Rev. D 91 (2015) 052007 [arXiv: 1407.1376] [INSPIRE].

[83] D. Hanneke, S.F. Hoogerheide and G. Gabrielse, Cavity Control of a Single-Electron Quantum Cyclotron: Measuring the Electron Magnetic Moment, Phys. Rev. A 83 (2011) 052122 [arXiv: 1009.4831] [INSPIRE].

[84] DELPHI collaboration, J. Abdallah et al., Study of tau-pair production in photon-photon collisions at LEP and limits on the anomalous electromagnetic moments of the tau lepton, Eur. Phys. J. C 35 (2004) 159 [hep-ex/0406010] [INSPIRE].

[85] Particle Data Group collaboration, K.A. Olive et al., Review of Particle Physics, Chin. Phys. C 38 (2014) 090001 [inSPIRE].

[86] D. Chang, W.-F. Chang, C.-H. Chou and W.-Y. Keung, Large two loop contributions to g- 2 from a generic pseudoscalar boson, Phys. Rev. D 63 (2001) 091301 [hep-ph/0009292] [INSPIRE].

[87] A. Dedes and H.E. Haber, Can the Higgs sector contribute significantly to the muon anomalous magnetic moment?, JHEP 05 (2001) 006 [hep-ph/0102297] [INSPIRE]. 
[88] K.-m. Cheung, C.-H. Chou and O.C.W. Kong, Muon anomalous magnetic moment, two Higgs doublet model and supersymmetry, Phys. Rev. D 64 (2001) 111301 [hep-ph/0103183] [INSPIRE].

[89] K. Cheung and O.C.W. Kong, Can the two Higgs doublet model survive the constraint from the muon anomalous magnetic moment as suggested?, Phys. Rev. D 68 (2003) 053003 [hep-ph/0302111] [INSPIRE].

[90] J.F. Gunion, A Light CP-odd Higgs boson and the muon anomalous magnetic moment, JHEP 08 (2009) 032 [arXiv:0808.2509] [inSPIRE].

[91] V. Ilisie, New Barr-Zee contributions to $(\mathbf{g}-\mathbf{2})_{\mu}$ in two-Higgs-doublet models, JHEP 04 (2015) 077 [arXiv: 1502.04199] [inSPIRE].

[92] J. Brod, U. Haisch and J. Zupan, Constraints on CP-violating Higgs couplings to the third generation, JHEP 11 (2013) 180 [arXiv:1310.1385] [INSPIRE].

[93] J.F. Kamenik, M. Papucci and A. Weiler, Constraining the dipole moments of the top quark, Phys. Rev. D 85 (2012) 071501 [Erratum ibid. D 88 (2013) 039903] [arXiv:1107.3143] [INSPIRE].

[94] G. Degrassi, E. Franco, S. Marchetti and L. Silvestrini, QCD corrections to the electric dipole moment of the neutron in the MSSM, JHEP 11 (2005) 044 [hep-ph/0510137] [INSPIRE].

[95] J. Hisano, K. Tsumura and M.J.S. Yang, QCD Corrections to Neutron Electric Dipole Moment from Dimension-six Four-Quark Operators, Phys. Lett. B 713 (2012) 473 [arXiv:1205.2212] [INSPIRE].

[96] E. Braaten, C.-S. Li and T.-C. Yuan, The Evolution of Weinberg's Gluonic CP-Violation Operator, Phys. Rev. Lett. 64 (1990) 1709 [INSPIRE].

[97] D. Chang, T.W. Kephart, W.-Y. Keung and T.C. Yuan, The Chromoelectric dipole moment of the heavy quark and purely gluonic CP-violating operators, Phys. Rev. Lett. 68 (1992) 439 [INSPIRE].

[98] M. Pospelov and A. Ritz, Electric dipole moments as probes of new physics, Annals Phys. 318 (2005) 119 [hep-ph/0504231] [inSPIRE].

[99] T. Bhattacharya, V. Cirigliano, R. Gupta, H.-W. Lin and B. Yoon, Neutron Electric Dipole Moment and Tensor Charges from Lattice QCD, Phys. Rev. Lett. 115 (2015) 212002 [arXiv: 1506. 04196] [INSPIRE].

[100] PNDME collaboration, T. Bhattacharya et al., Iso-vector and Iso-scalar Tensor Charges of the Nucleon from Lattice QCD, Phys. Rev. D 92 (2015) 094511 [arXiv:1506.06411] [INSPIRE].

[101] K. Fuyuto, J. Hisano and N. Nagata, Neutron electric dipole moment induced by strangeness revisited, Phys. Rev. D 87 (2013) 054018 [arXiv:1211.5228] [INSPIRE].

[102] D.A. Dicus, Neutron Electric Dipole Moment From Charged Higgs Exchange, Phys. Rev. D 41 (1990) 999 [INSPIRE].

[103] G. Blankenburg, J. Ellis and G. Isidori, Flavour-Changing Decays of a $125 \mathrm{GeV}$ Higgs-like Particle, Phys. Lett. B 712 (2012) 386 [arXiv:1202.5704] [INSPIRE].

[104] R. Harnik, J. Kopp and J. Zupan, Flavor Violating Higgs Decays, JHEP 03 (2013) 026 [arXiv:1209.1397] [INSPIRE]. 
[105] ETM collaboration, V. Bertone et al., Kaon Mixing Beyond the SM from $N_{f}=2$ tmQCD and model independent constraints from the UTA, JHEP 03 (2013) 089 [Erratum ibid. 1307 (2013) 143] [arXiv:1207.1287] [INSPIRE].

[106] ETM collaboration, N. Carrasco et al., B-physics from $N_{f}=2$ tmQCD: the Standard Model and beyond, JHEP 03 (2014) 016 [arXiv:1308.1851] [INSPIRE].

[107] N. Carrasco et al., $D^{0}-\bar{D}^{0}$ mixing in the standard model and beyond from $N_{f}=2$ twisted mass QCD, Phys. Rev. D 90 (2014) 014502 [arXiv:1403.7302] [inSPIRE].

[108] S. Davidson and G.J. Grenier, Lepton flavour violating Higgs and tau to mu gamma, Phys. Rev. D 81 (2010) 095016 [arXiv: 1001.0434] [InSPIRE].

[109] A. Goudelis, O. Lebedev and J.-h. Park, Higgs-induced lepton flavor violation, Phys. Lett. B 707 (2012) 369 [arXiv:1111.1715] [INSPIRE].

[110] M. Misiak and M. Steinhauser, NNLO QCD corrections to the $\bar{B} \rightarrow X_{s} \gamma$ matrix elements using interpolation in $m_{c}$, Nucl. Phys. B 764 (2007) 62 [hep-ph/0609241] [INSPIRE].

[111] M. Misiak et al., Updated NNLO QCD predictions for the weak radiative B-meson decays, Phys. Rev. Lett. 114 (2015) 221801 [arXiv:1503.01789] [INSPIRE].

[112] Heavy Flavor Averaging Group (HFAG) collaboration, Y. Amhis et al., Averages of b-hadron, c-hadron and $\tau$-lepton properties as of summer 2014, arXiv:1412.7515 [INSPIRE].

[113] S. Descotes-Genon, D. Ghosh, J. Matias and M. Ramon, Exploring New Physics in the $C_{7}-C_{7^{\prime}}$ plane, JHEP 06 (2011) 099 [arXiv:1104.3342] [INSPIRE].

[114] W. Altmannshofer and D.M. Straub, New physics in $b \rightarrow s$ transitions after LHC run 1, Eur. Phys. J. C 75 (2015) 382 [arXiv:1411.3161] [InSPIRE].

[115] A.J. Buras, M. Gorbahn, S. Jäger and M. Jamin, Improved anatomy of $\varepsilon / \varepsilon^{\prime}$ in the Standard Model, JHEP 11 (2015) 202 [arXiv:1507.06345] [INSPIRE].

[116] NA48 collaboration, J.R. Batley et al., A Precision measurement of direct CP-violation in the decay of neutral kaons into two pions, Phys. Lett. B 544 (2002) 97 [hep-ex/0208009] [INSPIRE].

[117] KTEV collaboration, A. Alavi-Harati et al., Measurements of direct CP-violation, CPT symmetry and other parameters in the neutral kaon system, Phys. Rev. D 67 (2003) 012005 [Erratum ibid. D 70 (2004) 079904] [hep-ex/0208007] [INSPIRE].

[118] KTEV collaboration, E.T. Worcester, The Final Measurement of $\varepsilon^{\prime} / \varepsilon$ from $K T e V$, in Heavy Quarks and Leptons 2008 (HQELLO8), Melbourne, Australia, 5-9 June 2008 [arXiv: 0909.2555] [INSPIRE].

[119] A.J. Buras, G. Colangelo, G. Isidori, A. Romanino and L. Silvestrini, Connections between epsilon-prime/epsilon and rare kaon decays in supersymmetry, Nucl. Phys. B 566 (2000) 3 [hep-ph/9908371] [INSPIRE].

[120] ATLAS collaboration, Search for $W^{\prime} \rightarrow t \bar{b}$ in the lepton plus jets final state in proton-proton collisions at a centre-of-mass energy of $\sqrt{s}=8 \mathrm{TeV}$ with the ATLAS detector, Phys. Lett. B 743 (2015) 235 [arXiv:1410.4103] [INSPIRE].

[121] ATLAS collaboration, Search for charged Higgs bosons in the $H^{ \pm} \rightarrow$ tb decay channel in $p p$ collisions at $\sqrt{s}=8 \mathrm{TeV}$ using the ATLAS detector, JHEP 03 (2016) 127 [arXiv: 1512.03704] [INSPIRE]. 
[122] LHCb collaboration, Measurement of Form-Factor-Independent Observables in the Decay $B^{0} \rightarrow K^{* 0} \mu^{+} \mu^{-}$, Phys. Rev. Lett. 111 (2013) 191801 [arXiv:1308.1707] [INSPIRE].

[123] LHCb collaboration, Angular analysis of the $B^{0} \rightarrow K^{* 0} \mu^{+} \mu^{-}$decay, LHCb-CONF-2015-002; CERN-LHCb-CONF-2015-002.

[124] LHCb collaboration, Test of lepton universality using $B^{+} \rightarrow K^{+} \ell^{+} \ell^{-}$decays, Phys. Rev. Lett. 113 (2014) 151601 [arXiv: 1406.6482] [INSPIRE].

[125] W. Altmannshofer and D.M. Straub, Implications of $b \rightarrow s$ measurements, in proceedings of the 50th Recontres de Moriond Electroweak interactions and unified theories, La Thuile, Italy, 14-21 March 2015, pp. 333-338 [arXiv: 1503.06199] [INSPIRE].

[126] R. Gauld, F. Goertz and U. Haisch, On minimal $Z^{\prime}$ explanations of the $B \rightarrow K^{*} \mu^{+} \mu^{-}$ anomaly, Phys. Rev. D 89 (2014) 015005 [arXiv: 1308.1959] [INSPIRE].

[127] W. Altmannshofer, S. Gori, M. Pospelov and I. Yavin, Quark flavor transitions in $L_{\mu}-L_{\tau}$ models, Phys. Rev. D 89 (2014) 095033 [arXiv:1403.1269] [INSPIRE].

[128] C. Niehoff, P. Stangl and D.M. Straub, Violation of lepton flavour universality in composite Higgs models, Phys. Lett. B 747 (2015) 182 [arXiv:1503.03865] [InSPIRE].

[129] S. Dawson and E. Furlan, A Higgs Conundrum with Vector Fermions, Phys. Rev. D 86 (2012) 015021 [arXiv:1205.4733] [INSPIRE].

[130] S. Fajfer, A. Greljo, J.F. Kamenik and I. Mustac, Light Higgs and Vector-like Quarks without Prejudice, JHEP 07 (2013) 155 [arXiv:1304.4219] [INSPIRE].

[131] R. Belusevic and J. Smith, $W-Z$ Interference in $\nu$-Nucleus Scattering, Phys. Rev. D 37 (1988) 2419 [INSPIRE].

[132] M. Cirelli, N. Fornengo and A. Strumia, Minimal dark matter, Nucl. Phys. B 753 (2006) 178 [hep-ph/0512090] [INSPIRE].

[133] E. Del Nobile, R. Franceschini, D. Pappadopulo and A. Strumia, Minimal Matter at the Large Hadron Collider, Nucl. Phys. B 826 (2010) 217 [arXiv:0908.1567] [InSPIRE].

[134] L. Di Luzio, R. Gröber, J.F. Kamenik and M. Nardecchia, Accidental matter at the LHC, JHEP 07 (2015) 074 [arXiv: 1504.00359] [INSPIRE].

[135] ATLAS collaboration, Search for pair and single production of new heavy quarks that decay to a $Z$ boson and a third-generation quark in pp collisions at $\sqrt{s}=8$ TeV with the ATLAS detector, JHEP 11 (2014) 104 [arXiv: 1409.5500] [INSPIRE].

[136] ATLAS collaboration, Search for vector-like B quarks in events with one isolated lepton, missing transverse momentum and jets at $\sqrt{s}=8 \mathrm{TeV}$ with the ATLAS detector, Phys. Rev. D 91 (2015) 112011 [arXiv:1503.05425] [INSPIRE].

[137] ATLAS collaboration, Analysis of events with b-jets and a pair of leptons of the same charge in pp collisions at $\sqrt{s}=8 \mathrm{TeV}$ with the ATLAS detector, JHEP 10 (2015) 150 [arXiv: 1504.04605] [INSPIRE].

[138] ATLAS collaboration, Search for production of vector-like quark pairs and of four top quarks in the lepton-plus-jets final state in pp collisions at $\sqrt{s}=8 \mathrm{TeV}$ with the ATLAS detector, JHEP 08 (2015) 105 [arXiv: 1505. 04306] [INSPIRE].

[139] ATLAS collaboration, Search for the production of single vector-like and excited quarks in the Wt final state in pp collisions at $\sqrt{s}=8 \mathrm{TeV}$ with the ATLAS detector, JHEP 02 (2016) 110 [arXiv: 1510.02664] [InSPIRE]. 
[140] CMS collaboration, Search for pair-produced vector-like B quarks in proton-proton collisions at $\sqrt{s}=8 \mathrm{TeV}$, arXiv:1507.07129 [INSPIRE].

[141] CMS collaboration, Search for vector-like charge $2 / 3$ T quarks in proton-proton collisions at $\sqrt{s}=8 \mathrm{TeV}$, Phys. Rev. D 93 (2016) 012003 [arXiv:1509.04177] [INSPIRE].

[142] Y. Gershtein et al., Working Group Report: New Particles, Forces and Dimensions, in Community Summer Study 2013: Snowmass on the Mississippi (CSS2013), Minneapolis, MN, U.S.A., July 29 - August 62013 [arXiv:1311.0299] [INSPIRE].

[143] Top Quark Working Group collaboration, K. Agashe et al., Working Group Report: Top Quark, in Community Summer Study 2013: Snowmass on the Mississippi (CSS2013), Minneapolis, MN, U.S.A., July 29 - August 62013 [arXiv:1311.2028] [INSPIRE].

[144] A. Falkowski, D.M. Straub and A. Vicente, Vector-like leptons: Higgs decays and collider phenomenology, JHEP 05 (2014) 092 [arXiv: 1312.5329] [INSPIRE].

[145] M. Son and A. Urbano, A new scalar resonance at $750 \mathrm{GeV}$ : Towards a proof of concept in favor of strongly interacting theories, arXiv:1512.08307 [INSPIRE]. 